\title{
Strongylides parasites de Mammifères du Congo-Brazzaville
}

\author{
(Collection R. Rousselot) ${ }^{1}$ \\ par Alassane DIAOURÉ \\ Vétérinaire-Inspecteur du Mali
}

\section{SOMMAIRE}

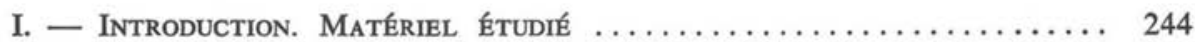

II. - Description des unités taxonomiques nouvelles $\ldots \ldots \ldots \ldots \ldots \ldots 246$

A. Agriostomum gorgonis Le Roux var. congolensis nov. ......... 246

B. Oesophagostomum rousseloti n. sp. ..................... 249

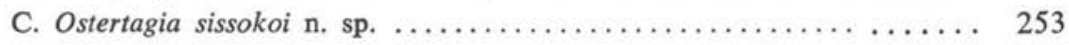

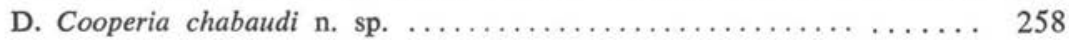

E. Ashworthius lerouxi n. sp. ....................... 262

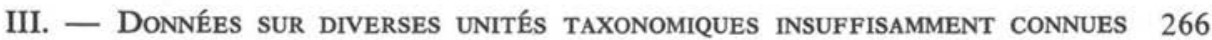

A. Redescription de Ternidens deminutus $=T$. simiae nov. syn. . . . 266

B. Espèces dont les échantillons congolais présentent quelques particularités 270

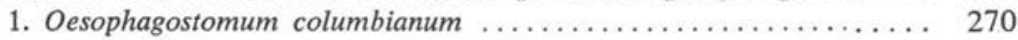

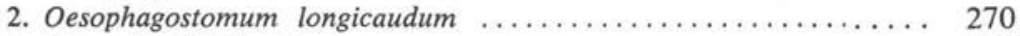

3. Cooperia fülleborni ........................... 270

C. Compléments iconographiques sur quelques espèces ........... 272

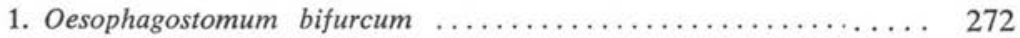

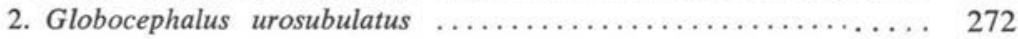

3. Cooperia pectinata .......................... 272

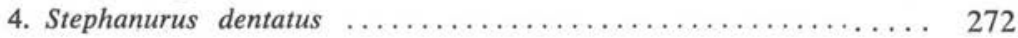

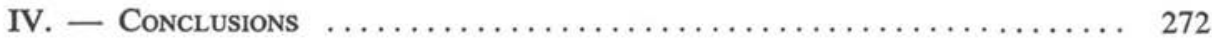

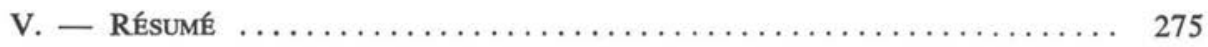

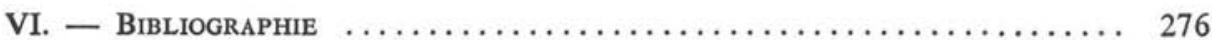

(1) Travail présenté pour l'obtention du diplôme de l'Ecole Pratique des Hautes-Etudes. 


\section{Introduction. Matériel étudié}

Au cours de notre stage au Laboratoire d'Helminthologie et Parasitologie Comparée de l'Ecole Pratique des Hautes-Etudes, le Professeur A.-G. Chabaud a bien voulu nous confier l'étude d'une collection de Strongylidés du Congo (Brazzaville). A ce propos nous exprimons au Professeur Chabaud et à tous nos maîtres de l'Université française, l'hommage de notre profonde gratitude.

Cette importante collection communiquée au Laboratoire de Zoologie (Vers) du Muséum National d'Histoire Naturelle par notre confrère R. Rousselot et dont l'étude complète nécessite plusieurs années nous a permis de nous familiariser avec des Helminthes tropicaux. Elle était à priori intéressante, car l'on ne possédait que peu de données sur les Nématodes des Mammifères domestiques et sauvages de cette partie de l'Afrique.

Nous avons identifié plusieurs espèces connues, décrit quatre espèces et une variété nouvelles, donné des figures complétant les descriptions classiques et mis Ternidens simiae Yamaguti, 1954 en synonymie de T. deminutus Raillet et Henry, 1905 après redescription de cette dernière espèce.

Nous donnons ci-dessous la liste des parasites examinés et de leurs hôtes ; certains parasites banaux ne sont indiqués que pour mémoire une fois pour toutes.

\section{STRONGYLOIDEA}

\section{SYNGAMIDAE.}

Stephanurinae.

Stephanurus dentatus Diesing, 1839, chez Sus scrofa domestica Gray, 1868.

\section{ANCYLOSTOMATIDAE.}

Globocephalinae.

Globocephalus urosubulatus Alessandrini, 1909, chez Sus scrofa domestica Gray, 1868.

\section{Uncinariinae.}

Bunostomum phlebotomum Railliet, 1900, chez Bibos indicus Linné, 1758.

Gaigeria pachyscelis Railliet et Henry, 1910, chez Syncerus nanus nanus Boddaert, 1785.

Ancylostomatinae Looss, 1905.

Agriostomum gorgonis Le Roux, var., congolensis nov., chez Kobus defassa Rüppel, 1835. 


\section{STRONGYLIDAE.}

Oesophagostominae.

Cyathostomum catinatum Looss, 1900. chez Equus caballus Linné, 1758.

Cyathostomum longibursatum Yorke et Macfie, 1918, chez Equus caballus Linné, 1758.

Cyathostomum nassatum Looss, 1900, chez Equus caballus Linné, 1758.

Cyathostomum goldi Boulenger, 1917, chez Equus caballus Linné, 1758.

Oesophagostomum (Proteracrum) columbianum (Curtice, 1890) Stossich., 1899, chez Ovis aries Linné, 1758.

Oesophagostomum (Bosicola) radiatum Rudolphi, 1803, chez Bos taurus Linné, 1758.

Oesophagostomum (Conoweberia) bifurcum Creplin, 1849, chez Mandrillus leucophaeus F. Cuvier, 1792.

Oesophagostomum (Oesophagostomum) longicaudum Goodey, 1925, chez Sus scrofa domestica Gray, 1868.

Oesophagostomum (Oesophagostomum) rousseloti n. sp., chez Sus scrofa domestica Gray, 1868.

\section{Strongylinae.}

Ternidens deminutus Railliet et Henry, 1905, chez Cercopithecus cephus Linné, 1758.

\section{TRICHOSTRONGYLOIDEA}

\section{TRICHOSTRONGYLIDAE.}

\section{Trichostrongylinae.}

Trichostrongylus colubriformis Giles, 1892, chez Ovis aries Linné, 1758

et

Ourebia ourebi dorcas Schwarz, 1914.

Ostertagia sissokoi n. sp., chez Sylvicapra grimmia Linné, 1758.

Cooperia fülleborni Hung, 1926, chez Sylvicapra grimmia Linné, 1758.

Cooperia pectinata Ransom, 1907, chez Bos taurus Linné, 1758.

Cooperia chabaudi, n. sp., chez Cephalophus dorsalis Gray, 1846.

\section{Nematodirinae.}

Haemonchus contortus Rudolphi, 1802, cbez Ovis aries Linné, 1758.

Ashworthius lerouxi n. sp., . chez Syncerus nanus nanus Boddaert, 1785. 


\section{METASTRONGYLIDAE.}

Metastrongylinae.

Metastrongylus ( = Choerostrongylus) pudendotectus Wostokow, 1905, chez Sus scrofa domestica Gray, 1868.

\section{Description des Unités taxonomiques nouvelles}

\section{A. - AGRIOSTOMUM GORGONIS Le Roux, 1929, var. CONGOLENSIS nov.}

(fig. 1 A-1 J)

Hôte: Kobus defassa Rüppel, 1835.

Origine : Région de Brazzaville (Congo).

Localisation : Intestin.

Matériel étudié : 3 우, 2 రై

\section{Description}

Caractères morphologiques généraux. - Vers longs, filiformes, blancs-grisâtres à cuticule très finement striée transversalement. La plus grande largeur est atteinte au niveau de la jonction de l'œsophage et de l'intestin. A partir de ce point le corps s'effile antérieurement et postérieurement. L'extrémité céphalique est cependant plus large que le cou. La bouche s'ouvre dorsalement. L'apex porte deux grosses amphides et quatre papilles médio-latérales apparemment doubles, les ventrales plus saillantes que les dorsales. La cavité buccale (fig. 1 C) comprend deux parties que sépare une formation annulaire: une partie proprement buccale avec une paroi dorsale presque inexistante et une paroi ventrale très développée, une partie œsophagienne en forme de cône à axe arqué dorsalement. La partie buccale dont la capsule est très chitinoïde possède une bouche triangulaire à angles arrondis; la paroi ventrale est haute de $0, z \mathrm{~mm}$ et la dorsale de $0,04 \mathrm{~mm}$. L'œsophage, dilaté en un grand infundibulum de $0,2 \mathrm{~mm}$ de base et $0,3 \mathrm{~mm}$ de profondeur, se rétrécit au niveau du cou, puis s'élargit après l'anneau nerveux. Le bord antérieur de la cavité buccale est armé de quatre paires de dents (fig. $1 \mathrm{~A})$; les dents antéro-ventrales sont petites et simples; les dents ventrolatérales, latérales et dorso-latérales situées respectivement en regard des papilles ventrales, des amphides et des papilles médio-dorsales, sont doubles et constituées par deux éléments superposés et inégaux, l'interne étant le plus petit. La dent dorsale

FIG. 1. - Agriostomum gorgonis var. congolensis. nov. A. Extrémité céphalique du mâle ; vue dorsale. B. Vue latérale gauche de l'extrémité antérieure de la femelle. C. Coupe longitudinale de l'extrémité céphalique du mâle; la dent ventrale n'est pas représentée sur cette moitié. D. Extrémité caudale et région vulvaire de la femelle. E. Vue latérale de la bourse caudale du mâle. F. Côtes dorsale et externo-dorsale. G. Vue latérale du cône génital du mâle. H. Cône génital, spicules et télamon (vue ventrale). I. Vue ventrale de la bourse copulatrice. J. Euf 

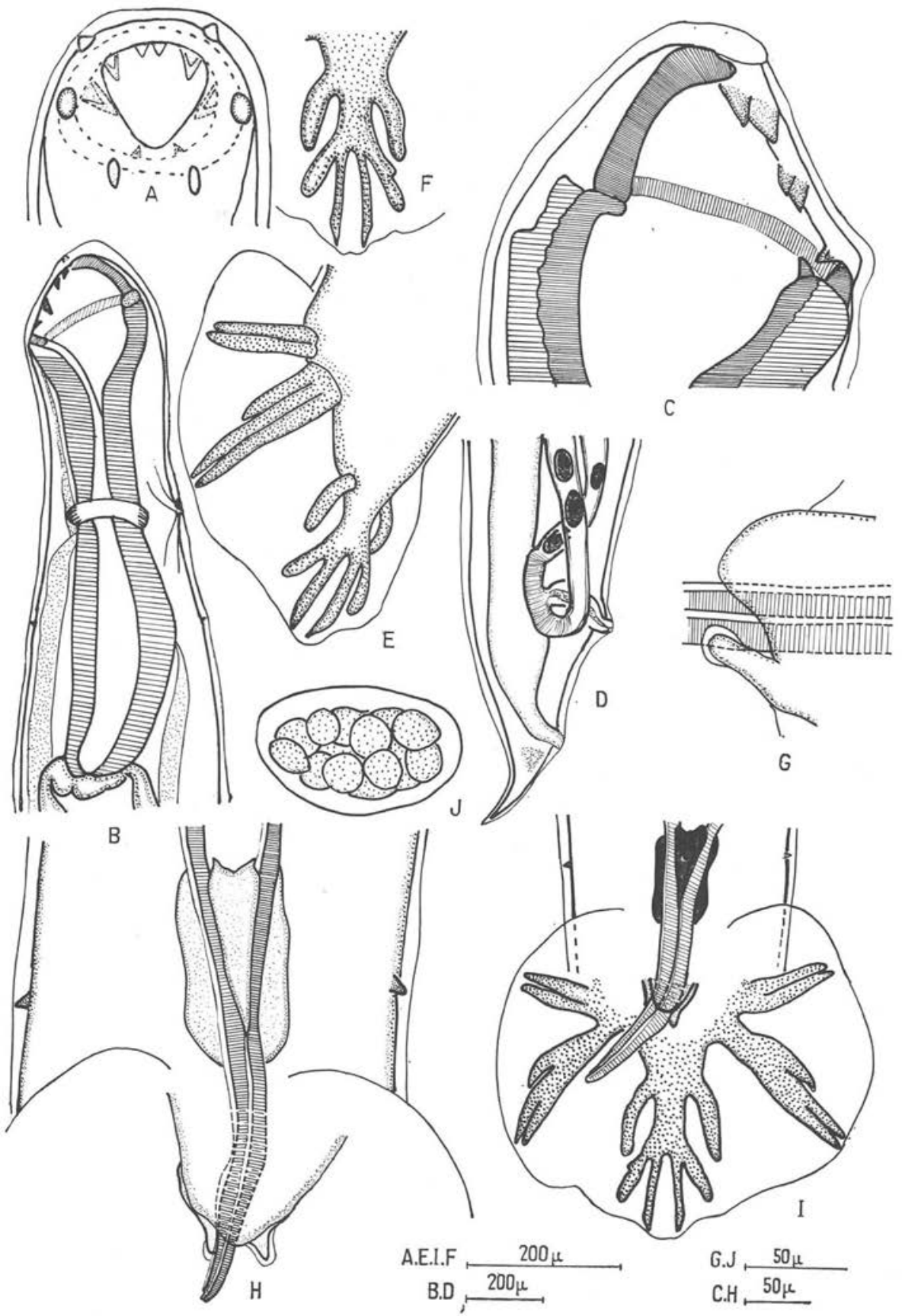
est très petite. Le bord interne de la capsule buccale porte un léger mamelon médioventral représentant un plateau coupant très réduit. L'anneau nerveux et le pore excréteur se situent vers le milieu de l'œsophage, les diérides vers le milieu du bulbe œsophagien. Le diamètre céphalique dorso-ventral est de $0,32 \mathrm{~mm}$ pour la femelle et $0,22 \mathrm{~mm}$ pour le mâle. Il n'existe ni ailes latérales, ni sillon cuticulaire ventral.

Femelle. - Longue de 11 à $14 \mathrm{~mm}$ sur $0,56 \mathrm{~mm}$ de large, elle présente un anneau nerveux, un pore excréteur et des diérides respectivement situés à $0,7 \mathrm{~mm}$, $0,7 \mathrm{~mm}$ et $0,98 \mathrm{~mm}$ de l'apex. L'anus est à $0,35 \mathrm{~mm}$ de la vulve et $0,3 \mathrm{~mm}$ de la pointe de la queue ; cette dernière est très pointue et légèrement recourbée vers le dos. L'œsophage mesure $1,5 \times 0,20-0,30 \mathrm{~mm}$. La vulve portée par une forte proéminence donne accès à un assez long vagin qui débouche à angle droit dans un ovéjecteur quasi-transversal. De ce dernier partent un utérus antérieur et un utérus postérieur qui dès sa naissance se recourbe en direction antérieure. L'œuf grossièrement ellipsoïde, contient une masse foncée de blastomères et mesure $0,088 \times 0,052 \mathrm{~mm}$.

Mâle. - Il mesure de 8 à $10 \mathrm{~mm}$. Sur le plus grand de nos spécimens, l'anneau nerveux et le pore excréteur sont à $0,5 \mathrm{~mm}$, les diérides à $0,72 \mathrm{~mm}$ de l'apex ; l'œsophage mesure $1,2 \times 0,15-0,20 \mathrm{~mm}$. La bourse caudale est symétrique. Les côtes ventrales subégales et adjacentes atteignent la marge bursale ; les côtes latérales naissent d'un tronc commun dont se détache d'abord l'antéro-latérale plus courte, plus grosse et n'atteignent pas les bords de la bourse comme les autres latérales. Le tronc commun à la dorsale et à l'externo-dorsale est épais à sa base ; juste avant son milieu il donne naissance à une grosse et courte externo-dorsale. La dorsale se dichotomise vers le tiers distal, presque au niveau de la terminaison de l'externo-dorsale, et chaque branche se divise aussitôt en deux autres : une interne fine, longue et à extrémité pointue, une externe grosse, plus courte, à extrémité arrondie et présentant parfois, à des niveaux variables, des tubercules. La partie dorsale du cône génital est caractérisée par l'existence de deux papilles latérales (fig. $1 \mathrm{H}$ ). Il n'existe pas de gubernaculum apparent. Par contre, les spicules sont maintenus par un télamon très peu chitinoïde constitué par une partie ventrale cordiforme et une partie dorsale formant deux branches latérales sur les bords externes des spicules. Ces derniers sont égaux, longs de 1,47 mm, filiformes et munis d'une aile membraneuse ; ils se dilatent vers l'extrémité distale avant de se terminer en pointe (fig. $1 \mathrm{H}$ ). Les papilles prébursales se situent à $0,1 \mathrm{~mm}$ de la base de la bourse.

\section{Discussion}

Ces Nématodes présentent tous les caractères du genre Agriostomum Railliet, 1902 et plus spécialement de l'espèce $A$. gorgonis Le Roux, 1929. Ils se distinguent cependant de cette dernière par :

- les dents de la paire dorsale beaucoup plus petites,

- un plateau coupant très réduit,

- une côte externo-dorsale plus courte se terminant en regard de la bifurcation de la dorsale,

- des spicules plus longs (1,47 au lieu de $1-1,20 \mathrm{~mm})$, et

- des diérides nettement en arrière de l'anneau nerveux. 
Ces différences paraissent d'autant plus faibles aue Le Roux a noté un polymorphisme assez important chez A. gorgonis. Cependant les hôtes sont assez éloignés, les aires géographiques ne coïncident pas et les caractères différentiels que nous signalons semblent constants sur tous nos spécimens. Nous préférons donc, à titre provisoire ne pas séparer spécifiquement les deux formes et nous proposons pour notre matériel, déposé au Muséum d'Histoire Naturelle à Paris sous le numéro $446 \mathrm{D}$, bocal N XXXVI, la désignation Agriostomum gorgonis var. congolensis nov.

\section{B. - OESOPHAGOSTOMUM ROUSSELOTI n. sp.}

(fig. $2 \mathrm{~A}-2 \mathrm{~F}, 3 \mathrm{~A}-3 \mathrm{~F}$ )
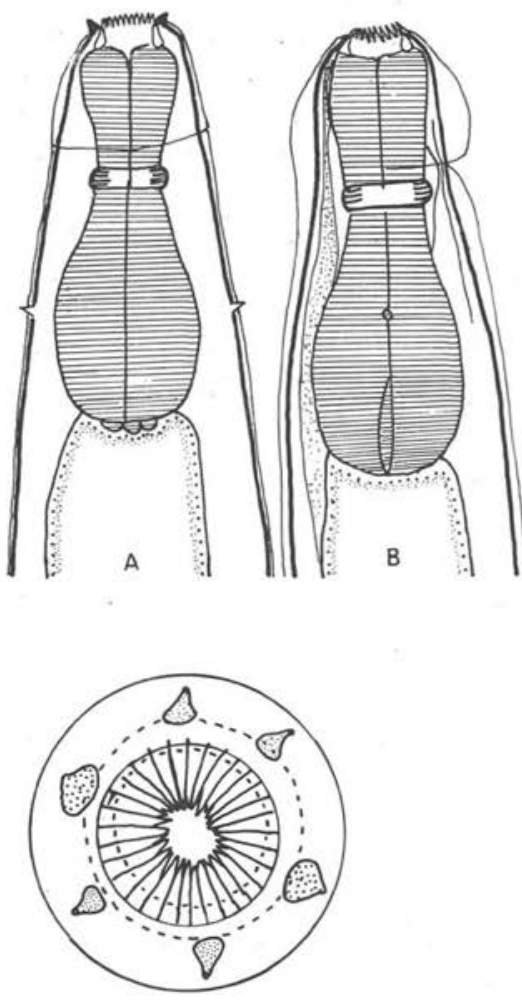

c

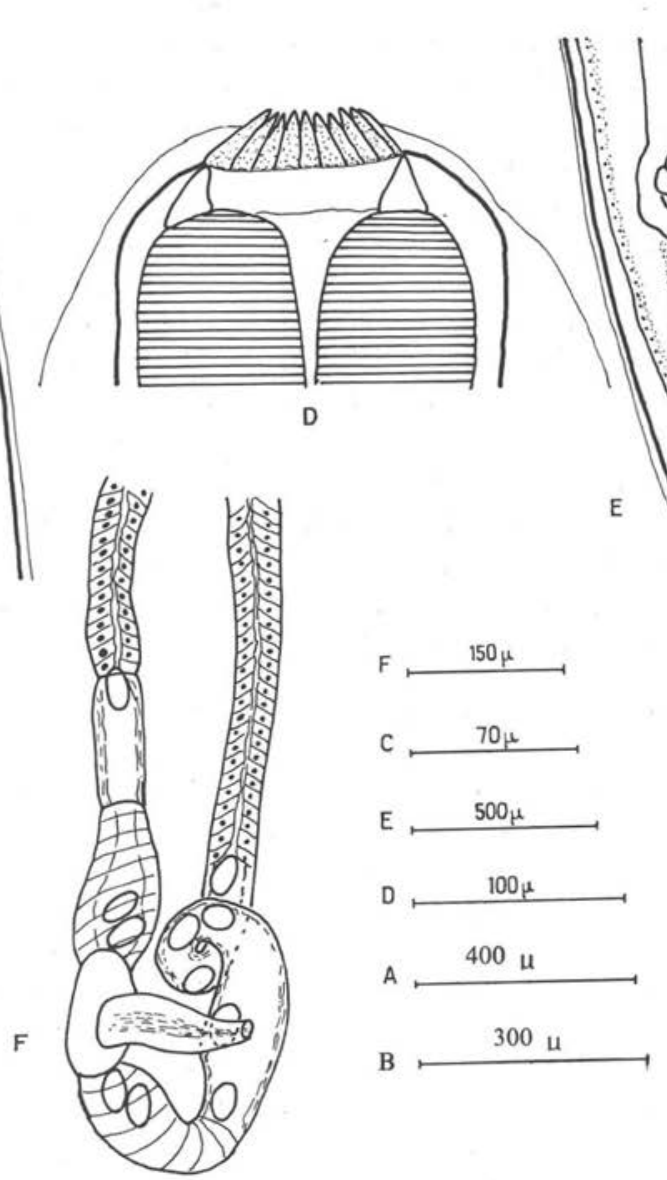

FIG. 2. - Esophagostomum rousseloti n. sp. A. Extrémité antérieure du mâle; vue ventrale. B. Extrémité antérieure du mâle; vue latérale. C. Vue apicale de l'extrémité céphalique. D. Extrémité antérieure au fort grossissement. E. Extrémite caudale de la femelle. F. Ovéjecteur 
RÉFÉRENCES BIBLIOGRAPHIQUes : Lane (C.), 1923 ; Le Roux (P.-L.), $1929 a$; Mönnig (H. O.), $1929 b, 1931 a, 1932 a$; Railliet (A.) et Henry (A.), 1913.

Hôte: Sus scrofa domestica Gray, 1868.

Origine : Région de Brazzaville (Congo).

Localisation: Côlon.

Matériel étudié : 4 우, 3 రై\%.

\section{Description}

Caractères morphologiques généraux. - Ces Nématodes, sont de taille relativement réduite, de couleur blanc-grisâtre foncé. L'ouverture buccale est nettement antérieure. L'extrémité céphalique présente une dilatation cuticulaire séparée du reste du corps par un sillon ventral ne s'étendant guère plus loin que les lignes latérales. Le pore excréteur se trouve au niveau de ce sillon, en avant de l'anneau nerveux. Les diérides, d'observation aisée, sont situées au niveau du grand diamètre de l'œsophage (un spécimen présente toutefois une diéride très antérieure). Ce dernier est en forme de massue fortement dilatée postérieurement, de diamètre légèrement plus grand antérieurement qu'au niveau de l'anneau nerveux. Il n'y a pas d'ailes latérales. L'extrémité apicale possède deux amphides latérales et quatre papilles submédianes plus petites. L'ouverture buccale circulaire est entourée d'une double «corona radiata ». Le cycle externe est constitué par 30 à 32 languettes à extrémité distale effilée ; le cycle interne comporte 60 à 64 éléments plus petits (le $1 / 3$ des externes), à extrémité distale plus arrondie. La capsule buccale, peu épaisse, est tronconique, à bords postérieurs plus épais et peu divergents ; son rapport hauteur/largeur est de $0,02 / 0,09 \mathrm{~mm}$, soit 1 pour 4,5 . Il n'existe pas de dents au fond de la cavité buccale.

La cuticule présente une fine striation transversale. Le diamètre céphalique est de $0,135 \mathrm{~mm}$ pour le mâle et $0,138 \mathrm{~mm}$ pour la femelle.

Femelle. - D'aspect effilé aux deux extrémités, elle mesure de 9 à $12 \mathrm{~mm}$ de long sur 0,6 mm de large. Sur une femelle de $10 \mathrm{~mm}$, l'œsophage mesure 0,6 $\times$ $0,27 \mathrm{~mm}$. Le sillon ventral, l'anneau nerveux, le pore excréteur et les diérides se situent respectivement à $0,20,0,23,0,25$ et $0,48 \mathrm{~mm}$ de l'extrémité apicale. La fente vulvaire peu visible est à $0,51 \mathrm{~mm}$ de l'anus qui se trouve à $0,41 \mathrm{~mm}$ de la pointe de la queue. Cette dernière est lisse, sans épines, s'effilant progressivement vers un bout arrondi. Le vagin long de $0,150 \mathrm{~mm}$ débouche dans une pars ejectrix de $0,120 \times$ $0,070 \mathrm{~mm}$ que prolonge, à chaque extrémité, un sphincter de $0,150 \times 0,080 \mathrm{~mm}$ renflé dans le tiers proximal; l'infundibulum cylindrique qui lui fait suite mesure $0,120 \times$ $0,50 \mathrm{~mm}$ et se prolonge par un oviducte à parois unicellulaires très épaisses. Ce long oviducte débouche dans un utérus à parois minces. L'utérus postérieur se courbe, aussitôt après le sphincter, en direction antérieure et, après un infundibulum allongé, décrit une boucle au niveau de l'oviducte avant de se prolonger vers l'avant de façon rectiligne. L'œuf, ellipsoïde, à paroi mince et à nombreux blastomères, mesure 0,052 $0,053 \times 0,032-0,033 \mathrm{~mm}$. 


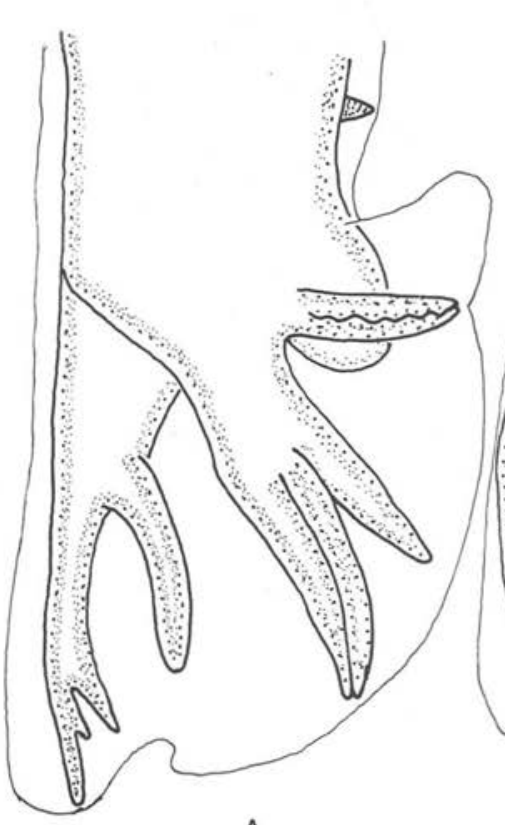

A

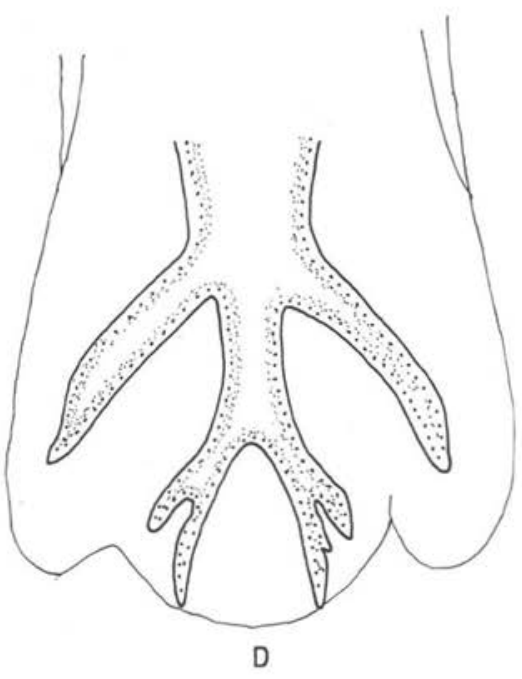

A.B.D $\longmapsto 200 \beta$
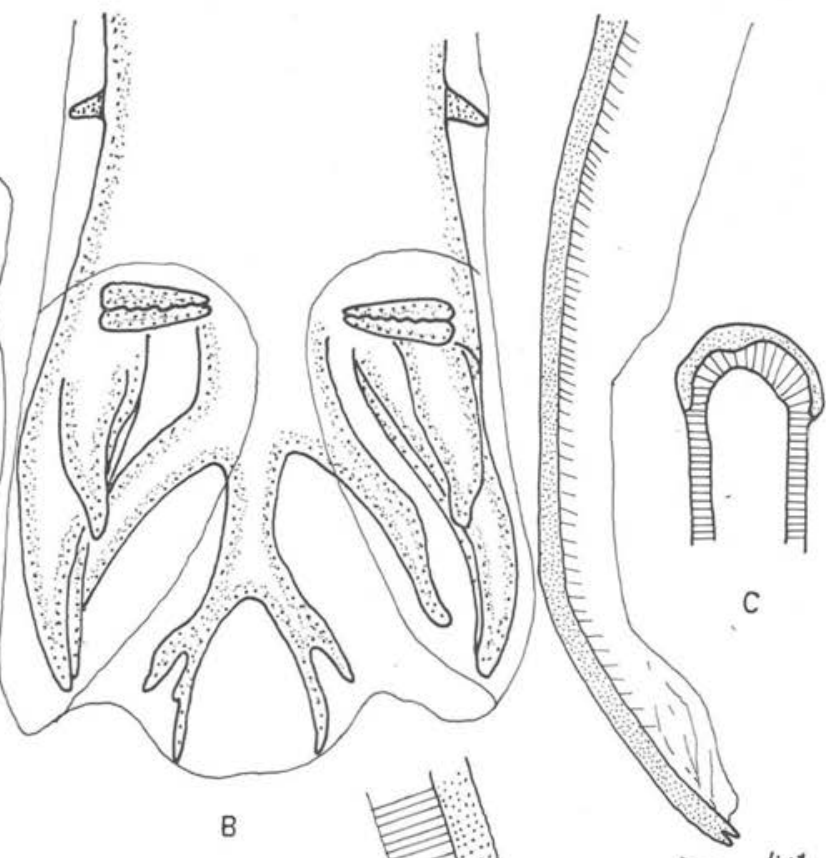

C

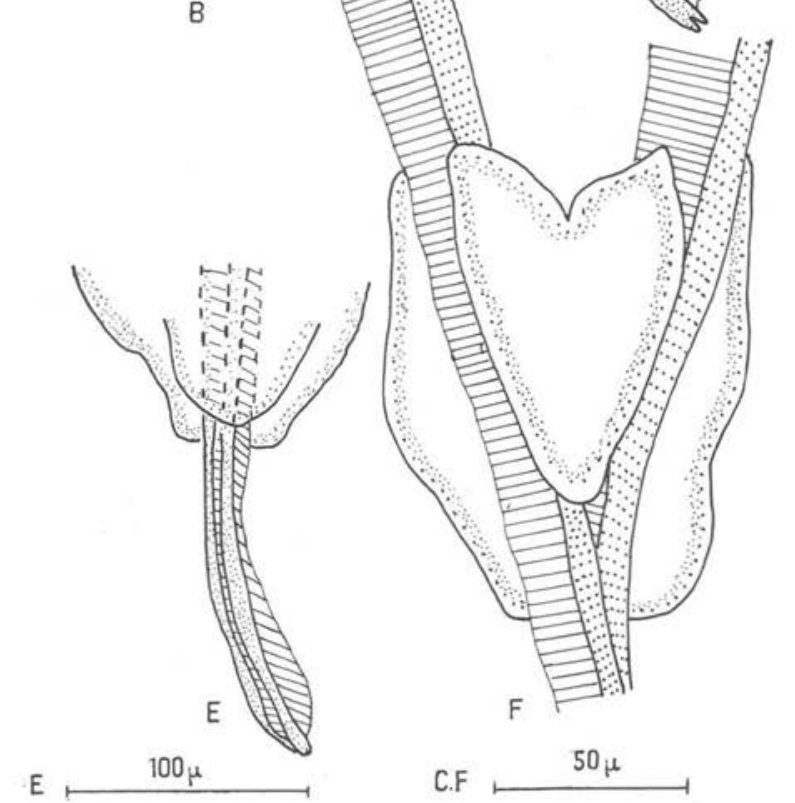

Fig. 3. - Esophagostomum rousseloti $n$. sp. A. Vue latérale de la bourse caudale. B. Vue ventrale de la bourse caudale. C. Extrémité distale et base d'un spicule. D. Côte dorsale. E. Cône génital et spicules. F. Détail du cône génital 
Mâle. - Il mesure 8,5-9 × 0,51 mm. Sur un mâle de $8,9 \times 0,51 \mathrm{~mm}$, l'œsophage mesure $0,52 \times 0,23 \mathrm{~mm}$; le sillon ventral, l'anneau nerveux, le pore excréteur et les diérides sont respectivement à $0,225,0,228,0,23$ et $0,45 \mathrm{~mm}$ de l'apex. La partie dorsale du cône génital est plus large que la ventrale. Les papilles prébursales, très apparentes, sont à $0,1 \mathrm{~mm}$ en avant de l'origine de la bourse caudale. Cette dernière, symétrique, est composée de deux lobes latéraux reliés par un lobe médian plus réduit. Les côtes ventrales parallèles et accolées atteignent les marges de la bourse; du tronc commun aux côtes latérales se détache une antéro-latérale plus courte que les autres et n'atteignant pas, comme elles, les bords du lobe, l'externo-dorsale, grosse et arquée, naît vers le milieu d'un tronc commun avec la dorsale. Cette dernière se dichotomise et chaque branche donne un rameau externe court et un rameau interne long, parfois orné d'une digitation accessoire. Les spicules, longs de $2,3 \mathrm{~mm}$, sont égaux, souvent recourbés dorsalement; ils sont filiformes et portent deux ailes membraneuses très développées. Un gubernaculum complexe les maintient solidement rendant leur dissection délicate à ce niveau. Ce gubernaculum se décompose en une partie ventrale cordiforme reliée, entre les spicules, à une partie dorsale plus évasée (fig. 3 F).

\section{Discussion}

Ce Nématode se classe dans le genre Oesophagostomum Molin, 1861. Il est plus spécialement caractérisé par le grand nombre d'éléments de sa double «corona radiata », sa petite taille et la longueur de ses spicules. Les espèces qui s'en rapprochent par les caractères des spicules ou par les éléments céphaliques s'en distinguent nettement par plusieurs caractères :

- Oe. (Daubneyia) mwanzae Daubney, 1924, parasite de Phacochoerus aethiopicus et Hippotragus equinus en Afrique, dont les spicules mesurent 2,075-2,9 mm selon Goodey, est une espèce plus grande dont la coronule ne comporte que six éléments externes et 14-15 internes.

- Oe. (Daubneya) oldi Goodey, parasite d'Hippotragus equinus et Phacochoerus aethiopicus en Afrique, dont les spicules sont longs de 2,4-2,6 mm, ne possède pas de coronule interne ; sa côte externo-dorsale naît près de la base de la dorsale et ses papilles pré-bursales sont peu visibles.

- Oe. (Proteracrum) hylochoeri Berghe, 1943, parasite d'Hylochoerus meinertzhageni au Parc National Albert, de taille voisine, possède des spicules plus longs $(3,162$ $3,187 \mathrm{~mm})$, une capsule buccale plus importante $(0,120 / 0,130)$, un vagin, une queue et des œufs plus longs, un œsophage et une vésicule céphalique plus courte. La coronule possède huit éléments externes et pas d'éléments internes.

- Oe. (Proteracrum) columbianum (Curtice, 1890) Stossich, 1899, parasite cosmopolite d'Ovis aries, qui possède une «corona radiata » à 20-24 éléments externes et 
40-48 internes, présente des spicules de 0,78-0,85 mm, des ailes latérales tout le long du corps et des diérides juste en arrière du sillon cuticulaire ventral.

- Oe. (Oesophagostomum) maplestoni (Schwartz, 1931), Schwartz 1932, possède bien 30 éléments externes, mais ses spicules ne mesurent que $0,832 \mathrm{~mm}$ et sa taille est plus petite $(6,87-6,91 \mathrm{~mm}$ pour le mâle, $8,2-9,1 \mathrm{~mm}$ pour la femelle).

- Oe. (Hudsonia) multifoliatum Daubney et Hudson, 1932, parasite de Capra hircus et Ovis aries, à 30 éléments externes et 60 internes possède en outre des ailes céphaliques bien développées, des spicules de 0,78-0,85 mm et un œuf long de 0,16 mm.

- Oe. (Hudsonia) walkeri Mönnig, 1932, parasite de Taurotragus oryx en Afrique, est pourvu de 25-28 éléments externes, 50-56 internes et a des ailes cervicales bien développées ; ses spicules ont seulement 0,787-0,84 mm de long ; l'œsophage est plus long $(0,82 \mathrm{~mm})$; le mâle mesure $12,35 \mathrm{~mm}$ et la femelle $18,3-20,3 \mathrm{~mm}$.

- Oe. (Oesophagostomum) africanum Mönnig, 1932, parasite d'Antidorcas troglodites en Afrique du Sud, possède également des ailes cervicales; ses spicules sont très réduits $(0,17-0,2 \mathrm{~mm})$; cette espèce se distingue donc de la nôtre malgré ses 28 31 éléments externes et 52-62 internes, par la taille de ses spicules et des spécimens (12,7-13,724 mm pour le mâle; 13,07-19 $\mathrm{mm}$ pour la femelle).

- Oe. (Oesophagostomum) aethiopicum Duthy, 1947, parasite de Phacochoerus aethiopicus au Tanganyika, de taille voisine (mâle de $8-9 \mathrm{~mm}$, femelle de 9,5-11,8 mm), à double corona de 26 éléments externes et 52 internes, possède des spicules plus courts $(1,307 \mathrm{~mm})$, un sillon cuticulaire beaucoup plus prononcé dorsalement, une capsule buccale plus petite et une côte externo-dorsale plus longue atteignant les marges bursales.

- Oe. watanabei Yamaguti, 1961, parasite de Sus leucomystax au Japon a bien une double corona de 28-31 éléments externes et 56-62 internes, un œsophage identique, un sillon ventral antérieur à l'anneau nerveux, des diérides au même niveau, mais possède par contre des œufs plus longs, des spicules plus petits $(0,83-0,9 \mathrm{~mm})$ et une côte postéro-latérale avec un rameau accessoire à sa base.

L'espèce étudiée se distingue donc des autres du genre, connues du porc et des hôtes voisins et nous proposons de la dénommer Oesophagostomum rousseloti $\mathrm{n}$. $\mathrm{sp}$. en hommage à notre confrère $\mathrm{R}$. Rousselot, Docteur-Vétérinaire à Brazzaville (Congo). Notre matériel est déposé au Laboratoire de Zoologie (Vers), Muséum National d'Histoire Naturelle à Paris sous les numéros $342 \mathrm{D}$ et $502 \mathrm{D}$, bocal N XXXVI.

RÉfÉrences bibliographiQues : Alicata (J. E), 1935 ; Baer (J. G.), 1950 ; Berghe (L. Van Den), 1943 ; Daubney (R.) et Hudson (J. R.), 1924, 1926, 1932 ; Duthy (B. L.), 1948 ; Goodey (T.), 1924 et 1925 ; Hung (See Lü), 1926; Leiper (R. T.), 1936 ; Le Roux (P.-L.), 1940 ; Ling (M. T.), 1959 ; Lizcano Herrera (J.), 1951 ; Maplestone (P. A), 1930 et 1931 ; Mönnig (H. O.), 1932 ; Railliet (A.), Henry (A. C. L.) et Bauche (J.), 1919 ; Sandground (J. H.), $1933 a, b$ et $c, 1937$. 


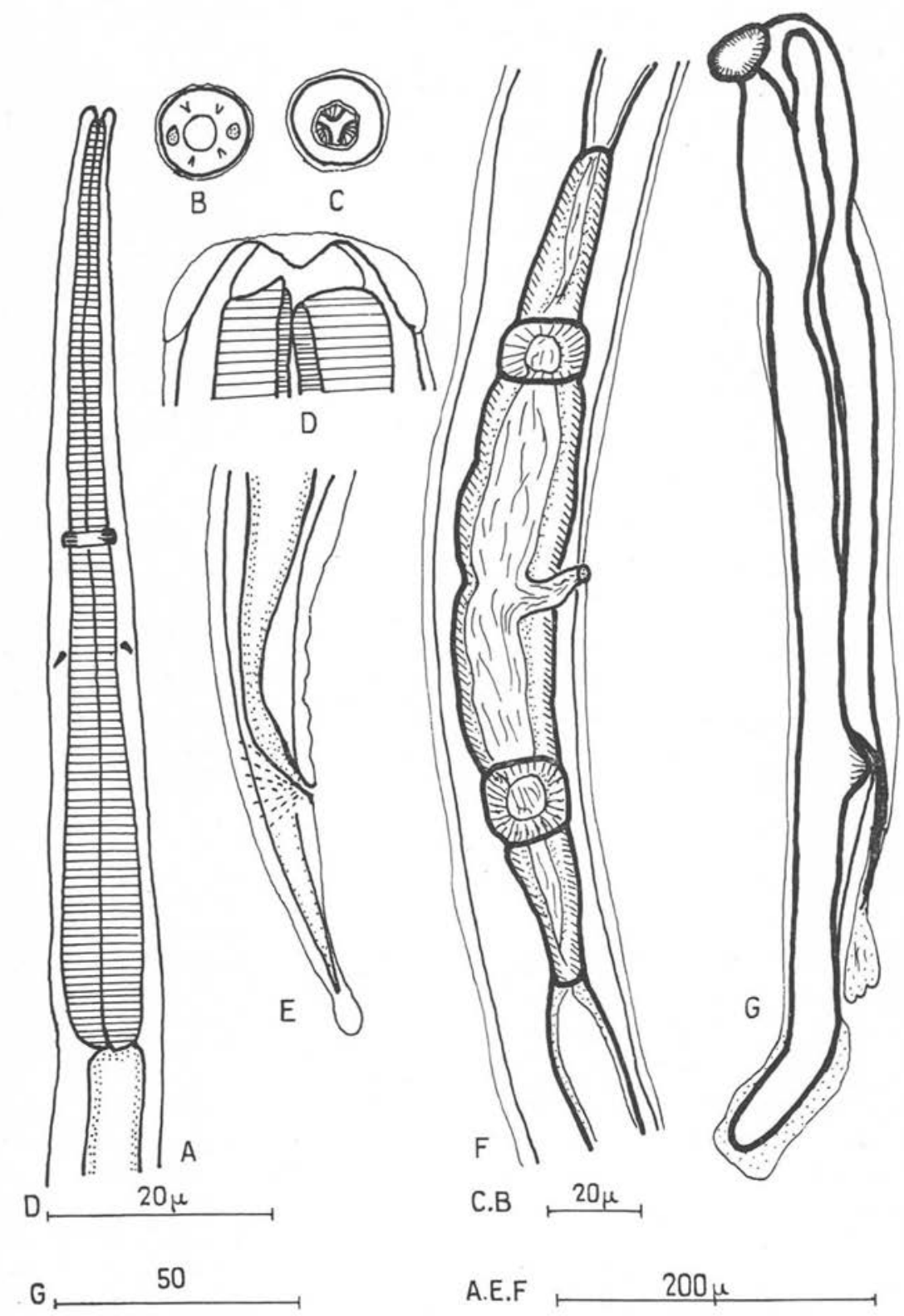

Fig. 4. - Ostertagia sissokoi n. sp. A. Extrémité antérieure de la femelle. B. Vue apicale. C. Vue apicale profonde. D. Extrémité céphalique au fort grossissement. E. Extrémité caudale de la femelle. F. Organes génitaux de la femelle. G. Spicule gauche 


\section{C. - OSTERTAGIA SISSOKOI n. sp.}

(fig. 4 A-4 G, 5 A-5 B)

Hôte : Sylvicapra grimmia Linné, 1758.

Origine: Parc Zoologique de Brazzaville (Congo).

Localisation : Caillette.

Matériel étudié : 2 우, 2 ఫో (femelles immatures).

\section{Description}

Caractères morphologiques généraux. - Nématodes filiformes de très petite taille (3,6 mm pour le mâle et $5,9 \mathrm{~mm}$ pour la femelle). Le corps est rétréci aux deux extrémités chez la femelle, à l'extrémité antérieure seulement chez le mâle. Il n'y a pas d'ailes latérales. La cuticule, très finement striée transversalement présente de nombreuses stries longitudinales (une vingtaine environ) d'un bout à l'autre du corps. L'ouverture buccale, circulaire, est entourée par deux amphides latérales et quatre papilles submédianes très fines. La cavité buccale, peu profonde, présente une section hexagonale en coupe optique. Les trois lobes œsophagiens occupent presque toute la cavité buccale sans dépasser le niveau de la bouche. L'œsophage s'élargit progressivement jusqu'à sa jonction avec un intestin simple et de diamètre uniforme. Dans la région du tiers moyen de l'œsophage se trouvent l'anneau nerveux, le pore excréteur et les diérides. Ces dernières, assez grandes, sont déportées vers la face dorsale du parasite. Le diamètre céphalique est de $0,020 \mathrm{~mm}$ en moyenne.

Femelle. - Sur la femelle de 5,9 $\times 0,13 \mathrm{~mm}$, l'œsophage mesure 0,52 $\times 0,05 \mathrm{~mm}$ l'anneau nerveux, le pore excréteur et les diérides sont respectivement situés à 0,27 , 0,32 et $0,35 \mathrm{~mm}$ de l'extrémité céphalique. La vulve est à $0,95 \mathrm{~mm}$ de l'anus qui se trouve à $0,15 \mathrm{~mm}$ de la pointe caudale.

Cette dernière est simple, sans épine, se terminant par un renflement cuticulaire. L'ouverture vulvaire transversale (fig. $4 \mathrm{~F}$ ), à bords très légèrement chitinoïdes, ne présente ni clapet, ni appendices. Le vagin est long de $0,04 \mathrm{~mm}$, l'ovéjecteur (fig. $4 \mathrm{~F}$ ) est constitué par les parties habituelles, chacune des deux branches étant longue au total de 0,55 mm. Les deux utérus sont opposés. Nos femelles étant immatures, il nous est impossible de donner les dimensions de l'œuf.

Mâle. - Sur le mâle de 3,6 mm, l'œsophage mesure 0,54 $\times 0,041 \mathrm{~mm}$. L'anneau nerveux, le pore excréteur et les diérides se situent respectivement à $0,255,0,31 \mathrm{~mm}$ de l'apex. La bourse caudale (fig. 5 A) symétrique est composée de deux larges lobes latéraux réunis par un lobe dorsal réduit et peu distinct. Les côtes ventrales, naissant d'un court tronc commun, courent latéralement, l'extrémité de la latéro-ventrale se courbant à angle droit vers l'extrémité de l'antéro-ventrale. Les côtes latérales naissent également d'un tronc commun dont la côte postéro-latérale se détache la première en direction postérieure tandis que les côtes antéro- et médio-latérales restent accolées sur la moitié de leur parcours avant de se séparer à angle aigu, la première latéralement et quelque peu en avant, la seconde en direction nettement postérieure. L'externo-dorsale longue 
et à base épaisse se détache indépendamment de la dorsale et suit un trajet à concavité intérieure. La dorsale se divise dans son tiers distal et chaque branche envoie une très courte ramification latérale avant de se terminer par une extrémité digitée. La bourse caudale parfaitement étalée est large de 0,35 et haute de $0,175 \mathrm{~mm}$. La côte dorsale, longue de $0,075 \mathrm{~mm}$, se dichotomise à $0,064 \mathrm{~mm}$ de sa base ; les fines papilles prébursales sont situées à $0,065 \mathrm{~mm}$ de la naissance de la bourse. Les spicules (fig. $4 \mathrm{G}$ ), plutôt trapus que filiformes, sont égaux et mesurent $0,250 \mathrm{~mm}$. Vers leur tiers distal, ils émettent une fine branche dorsale terminée par une expansion aliforme. Au même niveau on note la présence d'un tubercule arrondi. L'extrémité distale des spicules est en forme de pied humain qu'entoure une formation membraneuse.

L'examen sous différentes incidences et la dissection de la gaine des spicules n'ont pas mis en évidence la présence d'un gubernaculum. A la face interne de la bourse caudale, en arrière du cône génital, se trouve une membrane bursale accessoire bien visible (fig. $5 \mathrm{~B}$ ) comprenant une partie médiane et deux expansions latérales; la partie médiane présente deux épaississements symétriques dont chacun porte une papille distale.

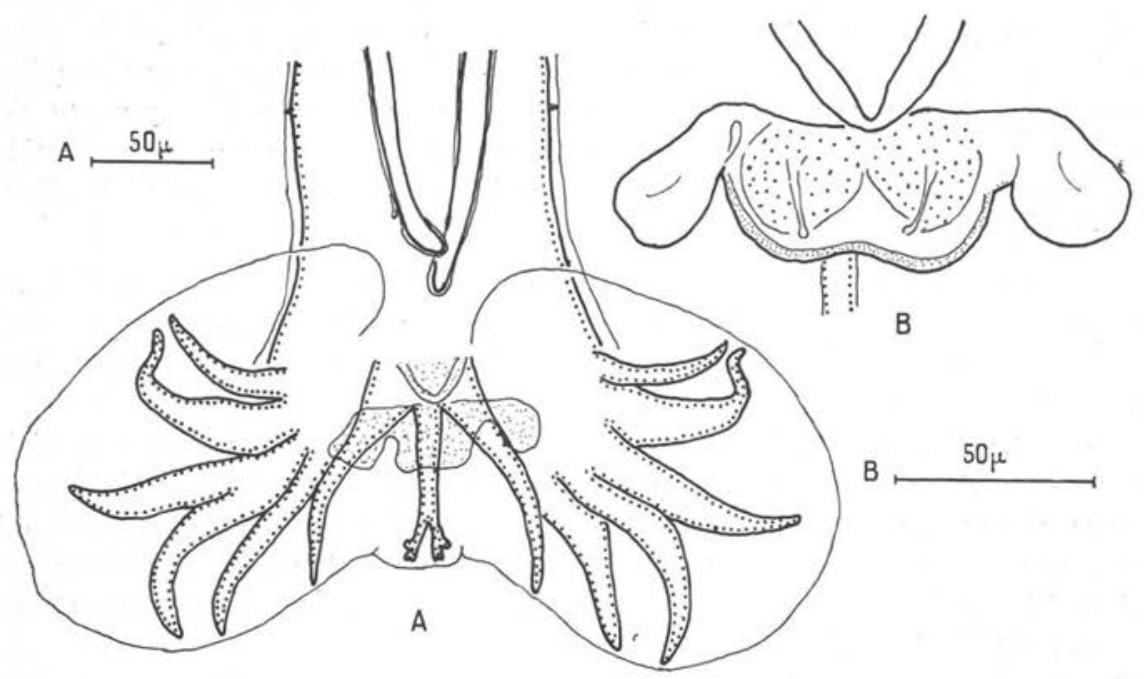

FIG. 5. - Ostertagia sissokoi n. sp. A. Vue ventrale de la bourse caudale. B. Cône génital et membrane bursale accessoire

\section{Discussion}

Ce Nématode Trichostrongylidae est un Trichostrongylinae du genre Ostertagia. Il paraît difficile de suivre la nomenclature complexe de Skrjabin et collaborateurs, 1954, car les espèces les plus proches de la nôtre possèdent un gubernaculum et se trouvent, de ce fait, placées dans des sous-genres différents. Nous prendrons le genre Ostertagia au sens large et examinerons les espèces particulièrement proches de la nôtre par la morphologie des spicules et de la bourse caudale : 
- O. mongolica Shumakovich, 1938, parasite de Capra hircus, Ovies aries, Camelus bactrianus et Procapra gutturosa en République Populaire Mongole, est une espèce plus grande (11,5-14 mm pour le mâle ; 13,87-17 $\mathrm{mm}$ pour la femelle). La côte dorsale mesure $0,310 \mathrm{~mm}$ et se bifurque avant son tiers distal ; les spicules ont 0,26-0,281 mm mais sont trifurqués.

- O. bisonis (Chapin, 1925) Kassimov, 1942, parasite de Bison bison en Amérique du Nord et au Canada, malgré un diamètre céphalique voisin $(0,019 \mathrm{~mm})$, une côte dorsale de même longueur et l'absence de gubernaculum se différencie par une côte dorsale bifurquée vers son milieu, une plus grande distance entre la côte médio-latérale et les côtes antéro- et postéro-latérales $(2 / 1$ au lieu de $3 / 1)$ et des spicules trifurqués mesurant 0,130-0,140 mm.

- O. popovi (Kassimov, 1942), Altaev, 1952, parasite d'Ovis aries en Russie, présente une côte dorsale plus longue $(0,164-0,171 \mathrm{~mm})$, un diamètre céphalique réduit $(0,014 \mathrm{~mm})$ et des spicules trifurqués.

- O. occidentalis Ransom, 1907 (=O. trifida Guillé, Marotel et Panisset, 1911) malgré une disposition semblable des diérides, de l'anneau nerveux et des côtes de la bourse, possède des spicules de $0,245-0,340 \mathrm{~mm}$, mais trifurqués, un gubernaculum nettement chitinoïde, un lobe dorsal plus long et une vulve dont l'ouverture est garnie de deux languettes saillantes. Cette espèce parasite la caillette et l'intestin grêle d'Ovis aries, Capra aegagrus, Capreolus capreolus, Ovis musimon et Ovis ophion armeniana en Asie, Russie et Amérique du Nord.

- O. trifurcata Ransom, 1907 (= O. pinnata Daubney, 1933), parasite d'Ovis aries, Capra hircus et Bos taurus en Europe occidentale, Asie, Afrique, et les deux Amériques, de Rangifer tarandus en Russie, de Pseudaxis nahura en nouvelle Zélande et de Damaliscus albifrons en Afrique, possède des spicules trifurqués. Les branches de la dorsale présentent un rameau externe, puis un rameau interne avant de se terminer en pointe unique, la côte ventro-latérale est presque parallèle à la ventro-ventrale.

- O. orloffi Sankin, 1930, parasite d'Ovies aries en U.R.S.S.. possède un gubernaculum bien visible, une côte dorsale divisée vers son milieu à angle plus ouvert et des spicules trifurquées mesurant 0,196-0,205 mm.

- O. polarica Pushmenkov, 1937, parasite de Rangifer tarandus en U.R.S.S., présente des spicules bifurqués, mais qui ne mesurent que $0,148 \mathrm{~mm}$ et possèdent une petite épine ; en outre, les côtes latérales antérieure et médiane ont même direction.

- O. mossi Dikmans, 1931, parasite d'Odocoileus virginianus aux U.S.A., présente des appendices périvulvaires, des spicules trifurqués et une côte dorsale ne se dichotomisant qu'une fois.

- O. leptospicularis Asadov, 1953, parasite de Capreolus capreolus en Azerbaidjan, ne présente aucune striation transversale; la côte dorsale donne, dans son tiers distal, deux branches dont chacune se termine par un rameau externe court et un rameau interne long. Les spicules, longs de $0,203-0,218 \mathrm{~mm}$, sont trifurqués. Le diamètre céphalique constant est de 0,012 . La femelle demeure inconnue.

- O. davtiani Grigoryan, 1951, parasite d'Ovis musimon et Capreolus capreolus en Arménie et Russie, présente un gubernaculum bien chitinoïde, des spicules bifurqués, 
une côte dorsale se dichotomisant vers le milieu de son trajet et des côtes ventrales parallèles et de grosseur équivalente.

- O. kolchida Popova, 1937, parasite de Bos taurus en Géorgie, présente des spicules trifurqués, un anneau nerveux et des diérides au niveau du tiers antérieur de l'œsophage.

- O. dahurica Orlov, Belova et Gnedina, 1931, parasite d'Ovis aries en Russie, présente un vagin dilaté à son origine, des spicules de 0,237-0,249 mm trifurqués, une côte dorsale de $0,102 \mathrm{~mm}$; la distance qui sépare les extrémités des côtes antéro-latérale et médio-latérale est supérieure à la distance entre l'antéro-latérale et la postéro-latérale (alors que les distances sont égales dans notre espèce).

- O. harrisi Le Roux, 1930, parasite de Tragelaphus sylvaticus en Afrique est l'espèce qui se rapproche le plus de la nôtre. Elle se différencie cependant par :

1. des spicules plus courts $(0,155-0,160 \mathrm{~mm})$,

2. une distance vulve-anus de $0,380-0,415 \mathrm{~mm}$ au lieu de $0,95 \mathrm{~mm}$,

3. la queue de la femelle retournée ventralement,

4. un utérus postérieur atrophié,

5. la présence d'un gubernaculum,

6. des expansions cuticulaires en avant de la vulve.

Nos échantillons se distinguent donc des autres Ostertagia et nous proposons le nom de Ostertagia sissokoi en hommage à notre confrère Sissoko Moussa, Directeur du Laboratoire du Service de l'Elevage et des Industries Animales à Bamako (République du Mali).

Nos spécimens sont déposés au Laboratoire de Zoologie (Vers) du Muséum National d'Histoire Naturelle à Paris sous le numéro $435 \mathrm{D}$, bocal N XXXVI.

Références bibliographiques : Altaev (A. K.), 1953) ; Andreeva (N. K.), 1958 ; Asadov (S. M.), 1954 ; Chapin (E. A.), 1925 ; Dikmans (G.), 1931 ; Gordon (H. Mc L.), 1932 ; Grigorian (G. A.), 1951 et 1952 ; Le Roux (P. L.), 1924 ; Orlov (I. V.), Belova et Gnedina (M. P.), 1931 et 1933 ; Popova (Z. G.), 1937 ; Pushmenkov (E. P.), 1937 ; Ransom (B. H.), 1907 et 1911 ; Sankin (S. L.), 1930.

$$
\text { D. - COOPERIA CHABAUDI n. sp. }
$$

(fig. 6 A-6 J)

Hôte: Cephalophus dorsalis Gray, 1846.

Origine: Parc Zoologique de Brazzaville.

Localisation: Intestin grêle.

Matériel étudié : 3 우, 1 бో.

\section{Description}

Caractères morphologiques généraux. - Nématodes filiformes mesurant $6,5 \mathrm{~mm}$ pour le mâle et $6,9-7,3 \mathrm{~mm}$ pour la femelle sur une largeur de 0,085-0,09 $\mathrm{mm}$. 


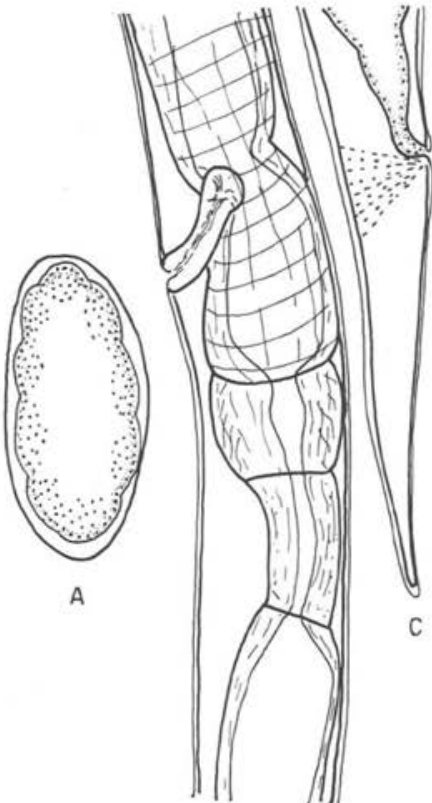

B

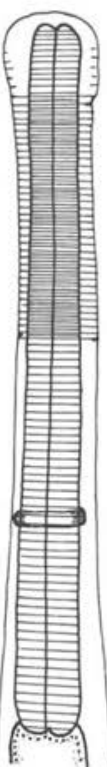

D

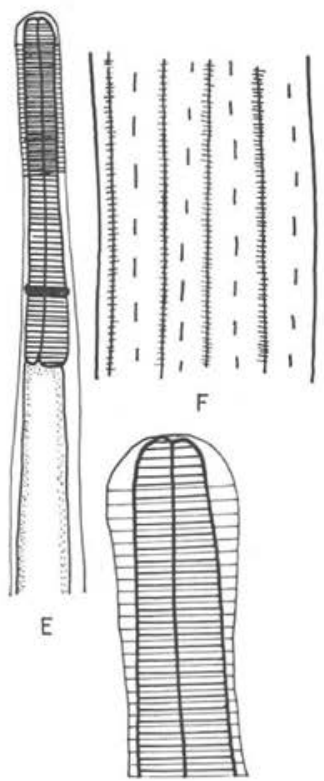

G

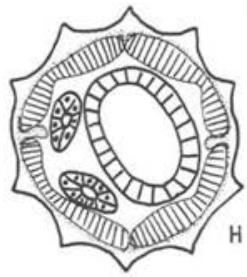

A.F.G.H.I.

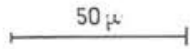

E.

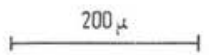

B.D.
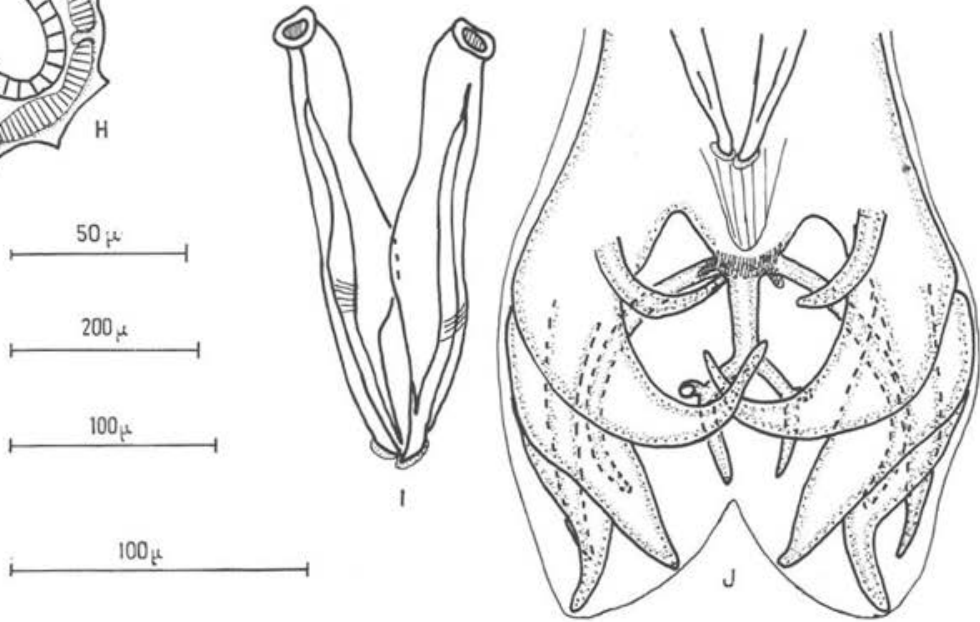

c.J.

$100 \mu$

Fig. 6. - Cooperia chabaudi n. sp. A. Euf. B. Ovéjecteur. C. Extrémité postérieure de la femelle. D. Extrémité antérieure de la femelle. E. Extrémité antérieure du mâle. F. Bandes cuticulaires longitudinales. H. Section transversale montrant les 10 crêtes longitudinales et 2 légères saillies au niveau des champs latéraux. I. Spicules. J. Bourse caudale du mâle 
La cuticule cervicale porte de profondes stries transversales s'étendant jusqu'à mi-hauteur environ de l'œsophage (fig. $6 \mathrm{D}$ ). Cette cuticule se dilate antérieurement en une vésicule céphalique (fig. $6 \mathrm{G}$ ), haute de $0,045 \mathrm{~mm}$ et large de $0,045 \mathrm{~mm}$; à son niveau les stries transversales ne sont bien visibles que latéralement. Les papilles et les amphides sont très peu discernables. Il n'existe ni ailes latérales ni diérides. L'anneau nerveux se situe au niveau du tiers inférieur de l'œsophage. Celui-ci se renfle postérieurement avant de s'aboucher à un intestin simple par le dispositif trivalvulaire habituel. La cavité buccale est rudimentaire et sa paroi très peu chitinoïde. Du dernier sillon cuticulaire transversal partent dix bandes longitudinales parallèles (fig. $6 \mathrm{~F}$ ) qui s'estompent quelque peu avant l'extrémité postérieure. Les crêtes saillantes qui les séparent sont très finement striées sur toute leur longueur (fig. $6 \mathrm{H}$ ). Le diamètre céphalique est de 0,032-0,043 $\mathrm{mm}$.

Femelle. - Sur une femelle mesurant $6,90 \times 0,085 \mathrm{~mm}$, l'œsophage est long de 0,35 et large de $0,035 \mathrm{~mm}$. Le diamètre céphalique est de $0,042 \mathrm{~mm}$. L'anneau nerveux est à $0,25 \mathrm{~mm}$ de l'apex. La vulve, à ouverture transversale très peu saillante et sans formations chitinoïdes, se situe dans le quart postérieur du Ver à $1,32 \mathrm{~mm}$ de la pointe caudale elle-même distante de $0,15 \mathrm{~mm}$ de l'anus. La queue (fig. $6 \mathrm{C}$ ) est pointue, sans épines. Le vagin long de $0,065 \mathrm{~mm}$ débouche dans un ovéjecteur long de 0,210 $\mathrm{mm}$. Les deux utérus (fig. $6 \mathrm{~B}$ ) sont divergents; l'utérus postérieur se recourbe dans la région préanale en direction antérieure. L'ovaire antérieur arrive presque à la jonction de l'œsophage et de l'intestin. Les œufs (fig. $6 \mathrm{~A}$ ) grossièrement ellipsoïdes mesurent $0,075-0,083 \times 0,038-0,045 \mathrm{~mm}$ et contiennent une masse foncée de blastomères.

Mâle. - L'unique mâle en notre possession mesure $6,5 \times 0,085 \mathrm{~mm}$; son œsophage $0,36 \times 0,04 \mathrm{~mm}$ et son diamètre céphalique est de $0,035 \mathrm{~mm}$. La bourse caudale (fig. $6 \mathrm{~J}$ ) symétrique est composée de deux grands lobes latéraux que réunit un petit lobe dorsal. Les côtes ventrales naissent séparément; la ventro-ventrale est fine et petite ; la latéro-ventrale qui représente, avec l'antéro-latérale, la plus grosse côte de la bourse, est épaisse à sa base, longue et fine à son extrémité distale recourbée en avant. Les côtes latérales naissent d'un tronc commun épais et court (fig. $6 \mathrm{~J}$ ) : l'antérolatérale est presque rectiligne, tandis que la médio-latérale se courbe en direction postérieure dans son tiers distal; contrairement aux autres latérales, la côte postérolatérale est fine et n'atteint pas les marges bursales.

L'externo-dorsale naît de la base de la dorsale; elle est moins large à sa base que la postéro-latérale qui, par ailleurs, est légèrement plus longue. La côte dorsale se divise vers le milieu de son trajet donnant deux branches en forme de lyre et se terminant chacune par une pointe unique ; chaque branche principale émet à la limite de son tiers antérieur et de son tiers moyen un rameau latéral arqué en dedans et en avant. Les spicules (fig. $6 \mathrm{I}$ ) longs de $0,13 \mathrm{~mm}$ sont renflés au milieu, ont un bord très peu pectiné et se terminent en bouton. Nous ne pouvons cependant préciser la structure intime de ces organes, car, nous avons préféré ne pas disséquer l'unique mâle dont nous disposons. Le cône génital apparaît comme une formation semi-circulaire avec deux papilles latérales coniques. Il n'existe ni gubernaculum, ni télamon, ni papilles pré-bursales discernables. 


\section{Discussion}

Bien que possédant dix bandes longitudinales, ce Trichostrongylidae est un Cooperia. Ransom indique 14-16 bandes dans sa définition générique de 1907 cependant que Cooperia reduncai de Brito Guterres, 1947 et Cooperia ershovi Wu, 1958 sont des espèces à dix bandes.

Le genre Cooperia compte, à notre connaissance, 22 espèces parmi lesquelles nous n'examinerons que les espèces se rapprochant de la nôtre par des caractères spécifiques importants tels que: longueur et structure des spicules et de l'ovéjecteur, longueur et conformation de la côte dorsale, dimensions et nombre de bandes longitudinales des spécimens.

- C. reduncai de Brito Guterres, 1947 a des spicules de 0,17 mm incisés sur leur ligne médiane et dix bandes longitudinales, mais c'est une espèce plus grande ( 8,8 et $9,2 \mathrm{~mm}$ pour les deux mâles décrits) et sa côte dorsale se bifurque dans son tiers distal.

- C. ershovi Wu, 1958 présente dix bandes longitudinales, mais ses spicules mesurent $0,249-0,420 \mathrm{~mm}$, ses trois côtes latérales sont de même grosseur, son externo-dorsale se sépare de la dorsale peu avant la bifurcation de cette dernière en deux branches parallèles portant des rameaux accessoires longs et rectilignes.

- C. memasteri Gordon, 1932, a une côte dorsale de 0,07-0,09 $\mathrm{mm}$ sur un mâle de $6,8 \mathrm{~mm}$, mais il possède 14 bandes longitudinales, un ovéjecteur de $0,67 \mathrm{~mm}$, des spicules de $0,27 \mathrm{~mm}$ bifurqués.

- C. yoshidai Mönnig, 1939, dont le mâle long de 7,2-9,8 mm possède des spicules de 0,134-0,152 mm, présente une côte dorsale à branches latérales naissant presque à sa bifurcation et deux rameaux accessoires près de son origine ; il existe des diérides à $0,38 \mathrm{~mm}$ de l'apex.

- C. laterouniformis Chen, 1937, malgré ses spicules de $0,138 \mathrm{~mm}$, son ovéjecteur de $0,3 \mathrm{~mm}$ est caractéristique par sa taille $(4,944 \mathrm{~mm}$ pour le mâle, $5,76 \mathrm{~mm}$ pour la femelle) et surtout par ses trois côtes latérales égales.

- C. curticei Giles, 1892 , bien que possédant des dimensions voisines $(5,41$ $7,27 \mathrm{~mm}$ pour le mâle, femelle de $6,57-7,38 \mathrm{~mm}$ ) et des spicules de 0,140-0,148 mm, se différencie de notre espèce par les branches latérales d'une dorsale longue de $0,09 \mathrm{~mm}$, mais à branches principales digitées distalement et à rameaux accessoires naissant très près de la bifurcation.

- $C$. borgesi de Brito Guterres, 1947, malgré un mâle de 6-7,5 $\mathrm{mm}$ et un ovéjecteur de $0,26 \mathrm{~mm}$ possède des spicules de $0,285-0,330 \mathrm{~mm}$ se terminant en pointe, une côte dorsale longue de $0,22 \mathrm{~mm}$ et 22 stries longitudinales.

- C. spatulata Baylis, 1938, dont le mâle mesure 6,3-7,5 mm possède deux bandes longitudinales, des diérides peu visibles, des branches latérales naissant en avant de la bifurcation de la dorsale; la côte antéro-latérale est la plus grosse de la bourse, l'ouverture vulvaire possède un clapet cuticulaire saillant; les spicules mesurent 0,23 $0,29 \mathrm{~mm}$ et l'ovéjecteur $0,3-0,55 \mathrm{~mm}$. 
- C. zurnabada Antipin, 1931, présente un mâle de 5,84-6,98 mm, un ovéjecteur de 0,39-0,592 mm, mais un anneau nerveux plus haut, douze bandes longitudinales, une queue plus longue $(0,174-0,212 \mathrm{~mm})$, des spicules longs de $0,228-0,235$ et bifurqués.

- $C$. oncophora Railliet, 1898, malgré un mâle de 6-8 $\mathrm{mm}$ et une hauteur des stries cuticulaires céphaliques voisines $(0,175-0,18 \mathrm{~mm})$ possède $12-15$ bandes longitudinales, une côte dorsale de 0,25-0,40 mm divisée dans son tiers postérieur en deux branches dichotomisées distalement ; la côte antéro-latérale est la plus grosse et l'externodorsale la plus longue; les spicules sont de 0,24-0,30 $\mathrm{mm}$; l'ovéjecteur est long de $0,25 \mathrm{~mm}$, la queue de la femelle est ornée de 7 à 11 stries transversales.

- C. fülleborni Hung, 1926, a des spicules de 0,165-0,196 mm et un mâle de 5,6-7,3 mm, mais présente un télamon, une longue côte externo-dorsale digitée distalement, des rameaux accessoires naissant à la bifurcation de la dorsale qui présente en outre deux minuscules branches accessoires près de la naissance de l'externo-dorsale ; il existe 14 bandes longitudinales ; la côte dorsale se bifurque dans son tiers antérieur.

- C. punctata Linstow, 1907, présente des spicules de 0,125-0,145 mm, une côte dorsale de 0,060-0,071 mm, un mâle de 5-9 $\mathrm{mm}$ et un ovéjecteur de $0,24 \mathrm{~mm}$, mais les branches accessoires se détachent presque en avant de la bifurcation de la dorsale en deux rameaux parallèles et digités distalement; les spicules présentent une très forte incision au milieu; la queue de la femelle est striée transversalement.

- C. minor de Brito Guterres, 1947, est l'espèce qui se rapproche le plus de nos spécimens par la longueur de ses spicules $(0,10-0,12 \mathrm{~mm})$ et de sa côte dorsale $(0,08 \mathrm{~mm})$, la largeur du mâle $(0,075-0,090)$. Elle s'en distingue cependant par un mâle de 3,5-4,8 mm, une femelle de 3,8-6,2 $\mathrm{mm}$ dont l'ovéjecteur mesure $0,01 \mathrm{~mm}$, des œufs plus petits $(0,065-0,075 \times 0,034-0,038 \mathrm{~mm})$, des spicules à base très large et tordue sur son axe, une côte ventro-ventrale plus longue, une côte dorsale à branches principales digitées distalement et présentant des rameaux accessoires en forme d'haltères juste à sa bifurcation et par son nombre plus élevé de bandes longitudinales (si l'on en juge par le dessin original).

Nos spécimens se distinguent donc de tous les autres Cooperia et nous proposons pour cette nouvelle espèce la dénomination Cooperia chabaudi en reconnaissance à notre maître le Professeur A. G. Chabaud, Directeur du Laboratoire de Zoologie (Vers) au Muséum National d'Histoire Naturelle à Paris où notre matériel est déposé sous le numéro $421 \mathrm{D}$, bocal N XXXV.

RÉfÉrENCES BIBLIOGRAPHIQUes: Antipin (D. N.), 1931 ; Baylis (H. A.), $1938 a$ et $b, 1939,1940$; Berghe (L. van den), 1937 ; Brito Guterres (J. de), 1946, 1947, 1952 ; Cameron (T. W. M.), 1939 ; Cram (E. B.), 1925 ; Gordon (H. Mc. L.), 1932 ; Hung (S. L.), 1926 ; Leiper (R. T.), 1911 b ; Le Roux (P. L.), 1930 b, 1936, 1950 ; Mönnig (H. O.), 1923, 1924, 1925, $1926 a, 1932$ b, 1939 ; Neveu-Lemaire (M.), 1936 ; Railliet (A.) et Henry (A. C. L.), $1909 a$; Ransom (B. H.), $1911 b$; Skrjabin (K. I.) et coll., 1954 ; Travassos (L. P.), 1921, 1937 ; Wu (S. C.), 1958 ; Yamaguti (S.), 1961 b ; Yorke et Maplestone, 1926. 


\section{E. - ASHWORTHIUS LEROUXI n. sp.}

(fig. $7 \mathrm{~A}-7 \mathrm{~K}$ )

Hôte: Syncerus nanus nanus Boddaert, 1785.

Origine: Brazzaville (Congo).

Localisation : Caillette.

Matériel étudié : 4 우, 3 \$ో\%

\section{Description}

Caractères morphologiques généraux. - Nématodes filiformes, allongés, mesurant $35-40 \mathrm{~mm}$ pour la femelle et $25-30 \mathrm{~mm}$ pour le mâle sur une largeur respective de 0,5 et $0,3 \mathrm{~mm}$. Il n'existe ni ailes latérales, ni dilatation céphalique de la cuticule. Cette dernière présente 29 à 30 stries longitudinales longues, mais peu épaisses. L'extrémité céphalique possède deux amphides et quatre grosses papilles médio-médianes en forme de croissants saillants; sa surface est formée de six lobes saillants subégaux. L'ouverture buccale est hexagonale et reliée aux saillies céphaliques par une mince membrane. La cavité buccale, profonde de $0,020 \mathrm{~mm}$, est délimitée par une très mince capsule buccale à section hexagonale et très légèrement asymétrique (fig. $7 \mathrm{H}$ ); son fond est armé d'une dent chitinoïde longue de $0,012 \mathrm{~mm}$ et fixée sur le lobe dorsal de l'œsophage. Ce dernier s'élargit progressivement jusqu'à sa jonction avec l'intestin. Le diamètre céphalique est de $0,032 \mathrm{~mm}$ en moyenne.

Femelle. - Sur une femelle mesurant $35 \times 0,5 \mathrm{~mm}$, l'anneau nerveux, le pore excréteur et les diérides se situent respectivement à $0,40 \mathrm{~mm}, 0,40 \mathrm{~mm}$ et $0,50 \mathrm{~mm}$. de l'extrémité apicale. Les diérides sont coniques et émergent d'une échancrure latérale de la cuticule; leur pointe est dirigée en arrière. L'œsophage est long de 1,65 mm et atteint sa largeur maximum de $0,2 \mathrm{~mm}$ à son extrémité distale.

L'anus est situé à $0,7 \mathrm{~mm}$ de la pointe de la queue. Celle-ci, lisse et simple ne présente pas d'épines. La vulve occupe une position variable, mais reste toujours située entre le quart et le tiers postérieurs du corps. La région vulvaire possède deux expansions cuticulaires aliformes subdorsales et deux languettes chitinoïdes de part et d'autre et légèrement en avant de la vulve (fig. $7 \mathrm{~F}$ et $7 \mathrm{~K}$ ), le vagin semble se contourner avant de déboucher dans la vaste pars éjectrix (nomenclature de Chitwood) qui présente à chacune de ses extrémités un léger rétrécissement et mesure $0,800 \times 0,150 \mathrm{~mm}$. Elle est suivie d'un sphincter de diamètre plus petit et long de $0,250 \mathrm{~mm}$; puis le diamètre s'accroît au niveau d'un infundibulum ovoïde qui débouche dans un utérus à paroi mince et rempli d'œufs. Les ovaires antérieur et postérieur naissent respectivement à 4 et $6 \mathrm{~mm}$ de l'apex, s'enroulent autour de l'intestin avant de se continuer jusqu'à l'utérus correspondant. L'œuf ellipsoïde (fig. 7 I) mesure $0,075 \times 0,045 \mathrm{~mm}$ et renferme une masse centrale à nombreux blastomères.

Mâle. - Chez un mâle de $30 \times 0,3 \mathrm{~mm}$, l'œsophage mesure $1,4 \times 0,125 \mathrm{~mm}$; l'anneau nerveux, le pore excréteur et les diérides sont respectivement situés à 0,35 , 0,35 et $0,43 \mathrm{~mm}$ de l'apex. Le cône génital (fig. $7 \mathrm{~J}$ ) est formé de deux languettes 


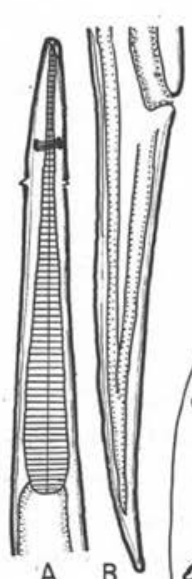

A
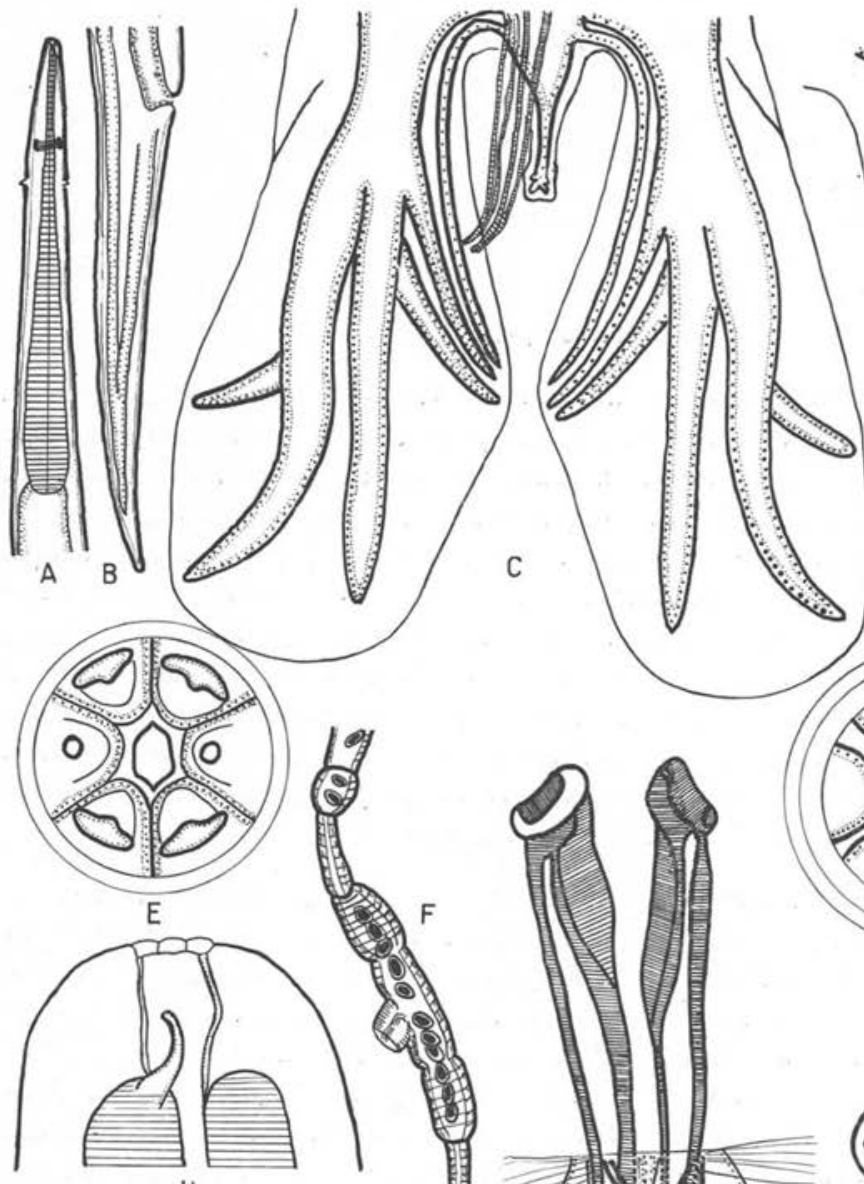

H
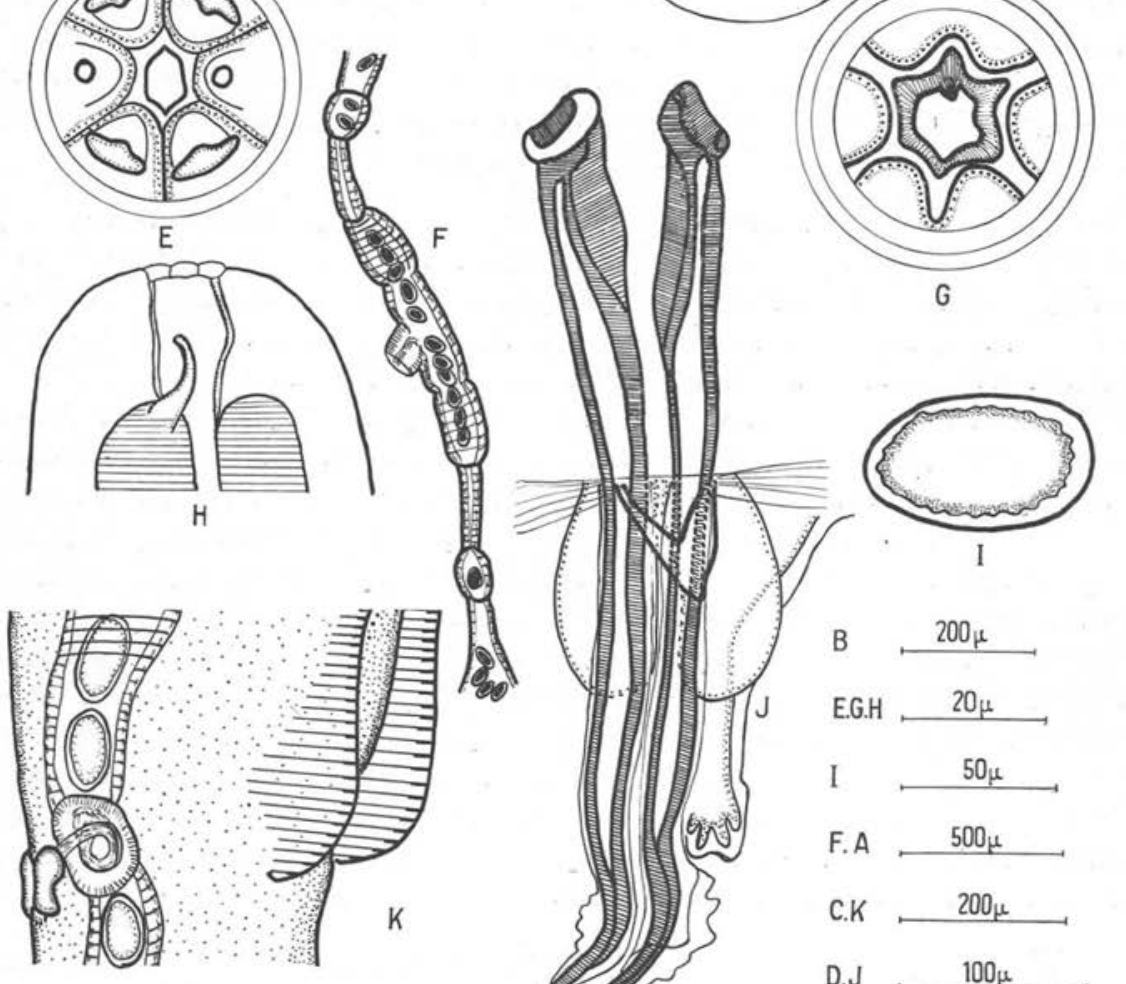

$$
\text { I }
$$

B

E.G.

$200 \mu$

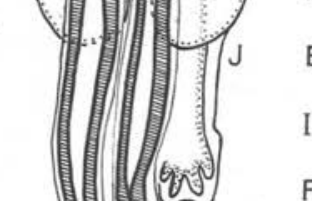

I

F. A

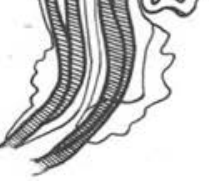

C.K

D.J

A $\quad 500 \mu$

C.K $200 \mu$

Fig. 7. - Asworthius lerouxi n. sp. A. Extrémité antérieure de la femelle. B. Extrémité postérieure de la femelle. C. Bourse caudale du mâle. D. Côte dorsale. E. Vue apicale de l'extrémité céphalique de la femelle. F. Ovéjecteur. G. Vue apicale profonde montrant l'unique dent œsophagienne. H. Extrémité apicale de la femelle montrant la capsule buccale et la dent œesophagienne. I. Euf. J. Spicules' cône génital et côte dorsale du mâle. K. Région vulvaire 
pyramidales dont la plus externe se termine par une papille et de deux formations en pied de bœuf à talon tronqué. Les spicules (fig. $7 \mathrm{~J}$ ) courts et trapus sont égaux; ils mesurent $0,5 \mathrm{~mm}$ de long pour une base de 0,049 et une partie moyenne de $0,012 \mathrm{~mm}$; ils sont plus fins vers leur extrémité qui se termine par une pointe légèrement renflée. Un étui membraneux crénelé à son extrémité distale recouvre chaque spicule ; les spicules ne présentent ni crochets ni épines sur toute leur étendue. Il existe deux petites papilles pré-bursales. La bourse caudale (fig. 7 C), parfaitement symétrique, se compose de deux grands lobes latéraux et d'un lobe médian atrophié représentant le quart ou le tiers des latéraux. Les côtes ventrales et latérales naissent d'un tronc commun et ne se séparent que vers le milieu de la ventro-ventrale; elles sont recourbées en avant. Du tronc commun aux latérales se détache d'abord la postéro-latérale, puis la médio-latérale qui se recourbent en arrière; l'antéro-latérale aussi développée que la latéro-ventrale est rectiligne. L'externo-dorsale très fine et longue naît presque indépendamment de la dorsale. Celle-ci est à grosse base et se dichotomise à angle aigu à son extrémité distale en deux éléments dont chacun se dichotomise à son tour presque aussitôt (fig. $7 \mathrm{D}$ ). Les lobes latéraux présentent des ornementations cuticulaires, en forme de massues, de croissants de lune, de points et de lames incurvées, spécialement marquées à la périphérie du lobe et entre les terminaisons des côtés.

\section{Discussion}

L'espèce décrite appartient à la famille des Trichostrongylidae Leiper, 1909. C'est un Nématodirinae Skrjabin et Orloff, 1914, puisqu'elle possède une dent œsophagienne absente chez les Trichostrongylinae Leiper, 1909 et les Molineinae Skrjabin et Schulz, 1937. Elle se distingue :

$1^{\circ}$ Des genres sans dent œsophagienne : Citellinema, Citellinoides, Longistrongylus, Obeliscoides, Travassostrongylus et Rinadia.

$2^{\circ}$ Des genres à côte dorsale séparée dès sa base et à vésicule céphalique : Nematodirus, Nematodirella.

$3^{\circ}$ De Mecistocirrus (Railliet et Henry, 1912), Neveu-Lemaire, 1914 à vulve antérieure, à spicules très longs, à côtes latéro-ventrale et ventro-ventrale très nettement séparées.

$4^{\circ}$ De Bohmiella, dont la cavité buccale, fortement chitinisée et en forme de V sur coupe transversale, est armée de cinq dents dont quatre petites; ses côtes latérales et externo-dorsale sont entourées d'une formation chitineuse. Il existe un gubernaculum.

Elle se rapproche plus spécialement des genres Haemonchus Cobb, 1898, Leiperiatus Sandgroung, 1930 et Asworthius Le Roux, 1930.

Haemonchus Cobb 1898 présente un gubernaculum et un lobe dorsal asymétrique.

Leiperiatus Sandground, 1930, parasite d'Hippopotamus amphibius possède une côte externo-dorsale plus courte, un œsophage divisé postérieurement, une ornementation vulvaire différente, une côte médio-latérale plus grosse que les autres latérales ; Sandground ne mentionne pas de diérides, cependant que Leiper en décrit. 
Ashworthius Le Roux, 1930, présente plusieurs caractères de nos spécimens : capsule buccale rudimentaire, cavité buccale petite et armée d'une grosse dent portée par le lobe dorsal de l'œsophage, petites diérides, bourse caudale du mâle avec deux lobes latéraux bien développés et un lobe dorsal réduit, côte ventro-ventrale environ deux fois moins longue et moins large que la latéro-ventrale avec laquelle elle possède un tronc commun; la côte antéro-latérale est la plus longue et la plus grosse des latérales et naît d'un tronc commun avec la médio-latérale, ce tronc commun annexant l'origine de la postéro-latérale; l'externo-dorsale est longue et parallèle à la postéro-latérale ; côte dorsale bien développée, se terminant par quatre digitations dont les deux internes sont les plus grosses; spicules relativement courts ; gubernaculum apparemment absent ; vulve dans la moitié postérieure du corps. Le genre compte actuellement trois espèces:

A. sidemi Schulz, 1933 et A. martinagliai Ortlepp, 1935, qui s'éloignent de la nôtre par leur côte dorsale à deux branches latérales vers le milieu de sa longueur.

A. pattoni Le Roux, 1930, qui présente une ouverture buccale nettement dorsale, un cône génital différent, une côte postéro-latérale plus longue, des spicules et une . ornementation vulvaire différents.

L'espèce décrite se distingue donc des trois Ashworthius connus. Nous pensons qu'elle est nouvelle et proposons de la dénommer Ashworthius lerouxi à la mémoire du regretté helminthologiste P. L. Le Roux. Notre matériel est déposé au Laboratoire de Zoologie (Vers), Muséum National d'Histoire Naturelle, Paris, sous le numéro 437 D bocal N XXXVI.

RÉfÉrences bibliographiQues : Chabaud (A. G.), 1959 ; Le Roux (P.-L.), 1929 b, 1930 b, Ortlepp (R. J.), 1935 ; Sandground (J. H.), 1930 ; Schulz (R. E. S.), 1933 ; Skrjabin et coll., 1954 ; Travassos (L. P.), 1921, 1937.

\section{Données sur diverses Unités taxonomiques insuffisamment connues}

On trouvera dans la présente section :

- la redescription de Ternidens deminutus Railliet et Henry, qui nous a paru d'autant plus nécessaire que nous considérons Ternidens simiae Yamaguti comme synonyme ;

- l'examen de quelques espèces dont les descriptions classiques ne s'appliquent pas tout à fait à nos échantillons, les différences constatées pouvant éventuellement relever de conditions propres au biotope forestier congolais ;

- le commentaire de plusieurs figures dont nous n'avons pas trouvé l'équivalent dans la littérature et que nous croyons utiles à la connaissance taxonomique des espèces correspondantes.

A. Redescription de TERNIDENS DEMINUTUS Railliet et Henry, 1905 (= T. Simiae Yamaguti, 1954) nov. syn. (fig. 8 A-8 I)

Hôte: Cercopithecus cephus Linné, 1758 (tube I). Simiens sp. (tubes 2 et 3). 
Origine: Parc Zoologique de Brazzaville (Congo).

Localisation : Intestin.

Matériel étudié : 12 exemplaires ( 8 우, $\left.4 \sigma^{\circ} \delta^{\star}\right)$.

Nous n'avons pas relevé de différences entre les spécimens des trois tubes. La description ci-dessous porte sur les spécimens du tube 1 .

Caractères morphologiques généraux. - Les spécimens mesurent 8,5-11,9 mm de long sur $0,48-0,60 \mathrm{~mm}$ de large pour les femelles et $9,2-10,5$ sur $0,45-0,51 \mathrm{~mm}$ pour les mâles. Le diamètre céphalique varie de 0,14 à $0,17 \mathrm{~mm}$. Vers longs, filiformes, de couleur blanchâtre après conservation dans l'alcool à 70 . Le corps est cylindrique, rétréci aux deux extrémités chez la femelle, à l'extrémité antérieure chez le mâle, il est finement strié transversalement. L'ouverture buccale est déportée vers la face dorsale et entourée d'une coronule de 20 à 23 éléments à bout libre plus ou moins arrondi (fig. $8 \mathrm{~A}$ et $8 \mathrm{~B}$ ). Après section longitudinale de l'extrémité céphalique d'un exemplaire, la coronule montre, à la base de chaque élément, des épaississements que certains auteurs décrivent comme une coronule interne. La tête porte deux grosses amphides latérales et quatre papilles médio-médianes plus fines. La capsule buccale est subglobuleuse et quelque peu comprimée dorso-ventralement; sa face interne est granuleuse (fig. $8 \mathrm{~A}$ ). Trois dents chitinoïdes portées par les lobes œsophagiens font saillie dans la cavité buccale une dorsale et deux ventro-latérales; chacune se compose de deux ailes incurvées vers l'axe du corps et d'une saillie médiane plus antérieure, l'ensemble présentant en coupe transversale l'aspect d'un $\omega$. L'œsophage, très peu dilaté à son origine, présente un goulot de diamètre uniforme, puis se dilate en forme de massue en arrière de l'anneau nerveux pour se terminer dans l'intestin par un dispositif trivalvulaire. L'intestin est de diamètre quasi uniforme sur toute sa longueur. L'animal ne présente ni ailes latérales, ni bourrelet péritomique, ni dilatation. Il existe un sillon cuticuticulaire ventral situé un peu en avant de l'origine de l'œsophage et dont les marges atteignent les lignes latérales. Le pore excréteur s'ouvre dans ce sillon. Les diérides et l'anneau nerveux se trouvent vers le milieu du goulot œsophagien. Le conduit de la glande œsophagienne dorsale s'ouvre très haut dans la capsule buccale.

Femelle. - Rétrécie aux deux extrémités, elle présente son maximum de largeur vers le milieu du corps. Le diamètre diminue très vite après la vulve, d'où une queue effilée, pointue, lisse et sans épines. Sur une femelle mesurant $11 \times 0,46 \mathrm{~mm}$, le pore excréteur, l'anneau nerveux et les diérides sont respectivement situés à $0,22,0,42$ et $0,42 \mathrm{~mm}$ de l'extrémité apicale. L'œsophage long de $0,72 \mathrm{~mm}$ possède une largeur maximum de $0,26 \mathrm{~mm}$ atteinte dans son cinquième postérieur. Les dents sont hautes de $0,085 \mathrm{~mm}$. La capsule buccale est haute de $0,21 \mathrm{~mm}$ et large de $0,17 \mathrm{~mm}$. Le sillon cuticulaire ventral se trouve à $0,16 \mathrm{~mm}$ de l'extrémité céphalique. La vulve, saillante, s'ouvre à $0,62 \mathrm{~mm}$ en avant de l'anus qui se trouve à $0,22 \mathrm{~mm}$ de la pointe caudale. Le vagin débouche dans un ovéjecteur légèrement asymétrique (fig. $8 \mathrm{D}$ ); les deux utérus remontent jusqu'en arrière du bulbe œsophagien, les deux ovaires descendent bas dans la région postérieure du corps. Les œufs ellipsoïdes (fig. $8 \mathrm{H}$ ) mesu- 


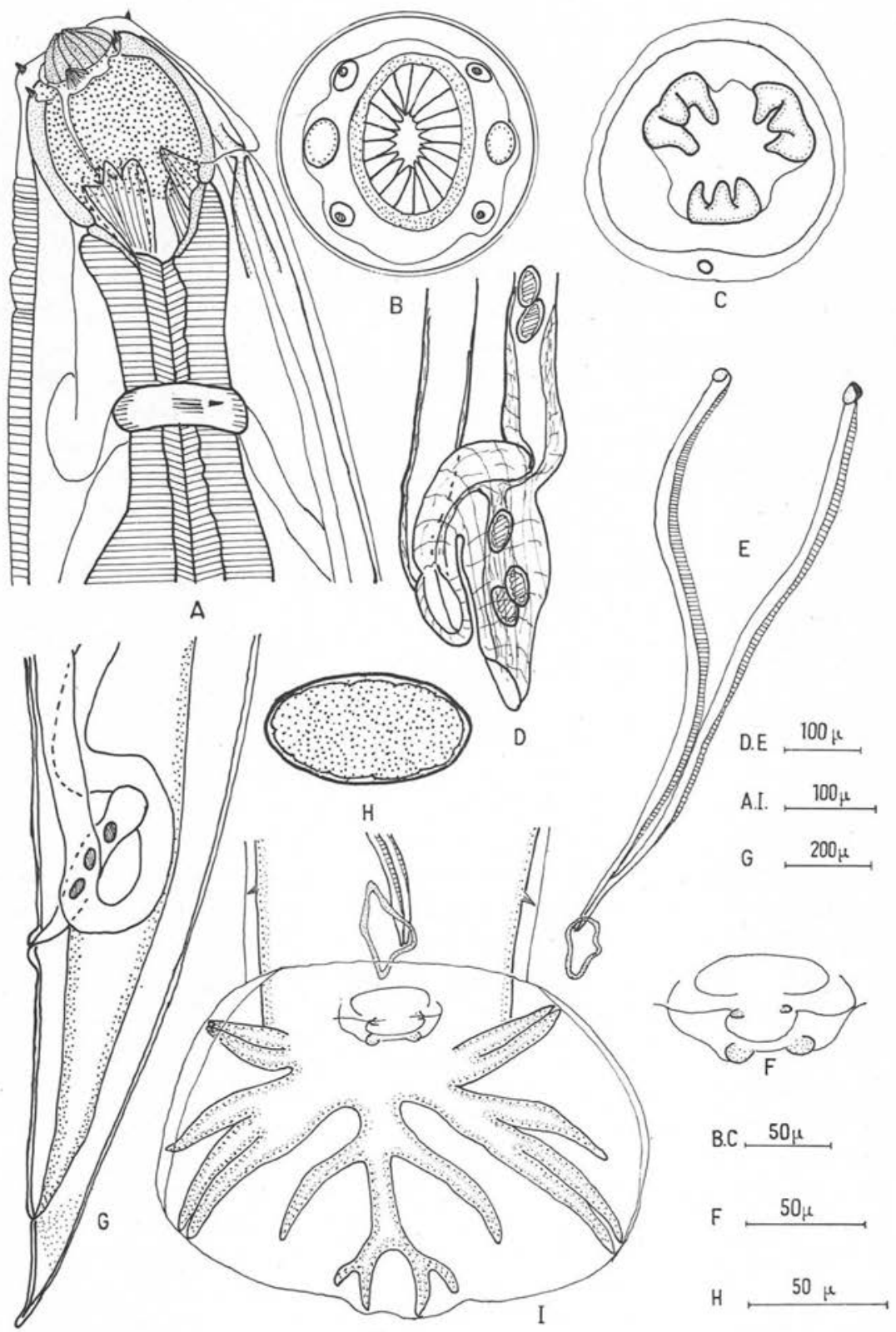

FIG. 8. - Ternidens deminutus Railliet et Henry, 1905. A. Extrémité céphalique de la femelle. B. Vue apicale. C. Vue profonde de la cavité buccale montrant les 3 dents à 3 éléments chacune. D. Ovéjecteur. E. Spicules. F. Cône génital mâle. G. Queue de la femelle. H. CEuf. I. Bourse caudale du mâle 
rent $0,062-0,072 \times 0,036-0,040 \mathrm{~mm}$; les blastomères sont en nombre extrêmement variable.

Mâle. - Il présente son maximum de largeur dans la moitié antérieure du corps. Sur un mâle de $10 \times 0,46 \mathrm{~mm}$, le pore excréteur, l'anneau nerveux et les diérides sont respectivement situés à $0,22,0,42$ et $0,42 \mathrm{~mm}$ de l'apex; l'œsophage mesure $0,70 \times$ $0,22 \mathrm{~mm}$; les dents sont hautes de $0,078 \mathrm{~mm}$; la capsule buccale mesure $0,20 \times$ $0,16 \mathrm{~mm}$. La bourse caudale symétrique, plus large que haute, est formée par deux grands lobes latéraux que réunit un petit lobe dorsal délimité par deux incisions peu profondes (fig. $8 \mathrm{I}$ ). Les côtes ventrales adjacentes atteignent presque les marges de la bourse. Les trois côtes latérales naissent d'un tronc commun; l'antéro-latérale s'en sépare la première ; elle est plus courte que les deux autres et, à la différence de celles-ci, n'atteint pas les bords de la membrane bursale. La côte externo-dorsale naît sur le tronc de la dorsale vers son tiers antérieur; la dorsale se divise en deux branches dont chacune se dichotomise à son tour en une courte branche externe et une branche interne qui atteint le bord du lobe dorsal. Les papilles pré-bursales sont situées à $0,055 \mathrm{~mm}$ de la naissance de la bourse. Le gubernaculum, de forme grossièrement naviculaire, mesure $0,11 \times 0,06 \mathrm{~mm}$. Les spicules longs de 1,15 à $1,17 \mathrm{~mm}$ selon les exemplaires, sont minces, égaux, ailés sur toute leur longueur (fig. $8 \mathrm{E}$ ), leurs extrémités distales sont accolées par les ailes.

\section{Discussion}

Ces Nématodes présentent tous les caractères de genre Ternidens Railliet et Henry, 1905 et, notamment de T. deminutus Railliet et Henry, 1905 (= Globocephalus macaci Smith, Fox et White, 1908). Aucune des différences entre les mensurations de nos échantillons et celles que rapportent les auteurs ne nous ayant paru significative (cf. tableau ci-dessous), nous rapportons nos échantillons à cette espèce.

Une seconde espèce, T. simiae Yamaguti, 1954, a été décrite dans le genre. Ses dimensions ne présentent pas non plus de différences significatives avec celles du type (cf. tableau ci-dessous).

En dehors de ses mensurations, Yamaguti caractérise son espèce par l'existence de dents à trois éléments qu'il oppose aux dents à deux éléments que les traités classiques attribueraient à $T$. deminutus.

En fait, sur une vue apicale de Globocephalus macaci $(=T$. deminutus $=$ Triodontophorus deminutus Railliet et Henry, 1905), Smith, Fox et White, 1908 (pl. VIII, fig. 1-2) figurent des dents à trois éléments et qui présentent en coupe optique l'aspect d'un $\omega$.

Railliet et Henry (1905) avaient déjà précisé : «Chacune de ces dents représente un angle dièdre dont l'arête regarde l'axe du corps : son bord supérieur n'est pas denticulé, mais forme simplement deux saillies arrondies séparées par une incision qui correspond à l'arête. » En outre, en 1912, lors de leur redescription de $T$. deminutus récolté sur Primates, ces auteurs écrivent: «Chacune de ces dents représente un 
angle dièdre dont l'arête, pourvue d'une carène, regarde l'axe du corps ", et figurent (pl. XXIV, fig. 2 et 3) une vue latérale et une vue interne d'une dent, ce qui met bien en évidence la présence de trois éléments par dent.

Les caractères des dents ne semblent donc pas différents chez $T$. deminutus Railliet et Henry, 1905 et chez T. simiae Yamaguti, 1954 et nous pensons qu'en conséquence on peut admettre leur synonymie.

MENSURATIONS COMPARÉES DE \& DIVERS 》 Ternidens

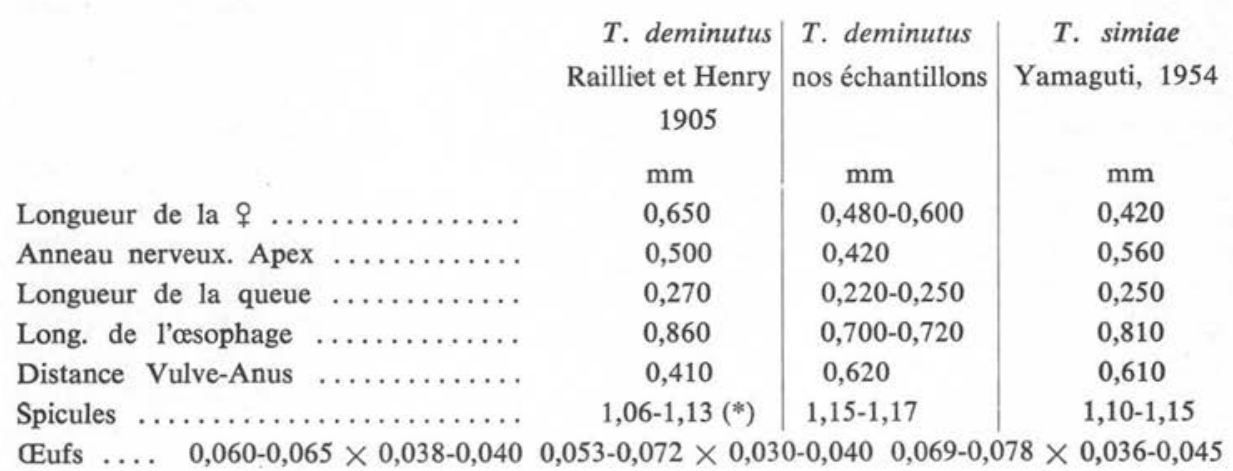

(*) D'après Skrjabin 1931 sur échantillons de Railliet et Henry.

\section{B. - Espèces dont les échantillons congolais présentent quelques particularités}

1. Oesophagostomum (Proteracrum) coliambianum (Curtice, 1890) Stossich, 1899. Comparés aux échantillons typiques tels qu'ils sont décrits par Stossich, nos mâles sont longs de $20-22 \mathrm{~mm}$ au lieu de $15-18 \mathrm{~mm}$. Les œufs sont moins larges $(0,040 \mathrm{~mm}$ au lieu de $0,050-0,060 \mathrm{~mm})$ bien que les femelles aient la même longueur $(12-14 \mathrm{~mm})$; les spicules sont longs de $0,75 \mathrm{~mm}$ au lieu de $0,78-0,85 \mathrm{~mm}$. Nous donnons (fig. $9 \mathrm{~A}$ ) une vue apicale de cette espèce, qui confirme le nombre de lamelles vues par Goodey.

2. Oesophagostomum (Oesophagostomum) longicaudum Goodey, 1925. Nous avons noté chez nos femelles une queue de 0,38 contre 0,453-0,543 mm chez les spécimens sud-africains de Goodey. Nous donnons une vue apicale (fig. $9 \mathrm{C}$ ).

3. Cooperia fïlleborni Hung, 1926. Nos femelles sont plus grandes $(9,4-10,5$ contre $5,4-7,8 \mathrm{~mm}$ ), les spicules ont $0,185 \mathrm{~mm}$ (au lieu de $0,165-0,195$ ) et le diamètre céphalique très légèrement plus petit $(0,035$ contre $0,040-0,050 \mathrm{~mm})$. 

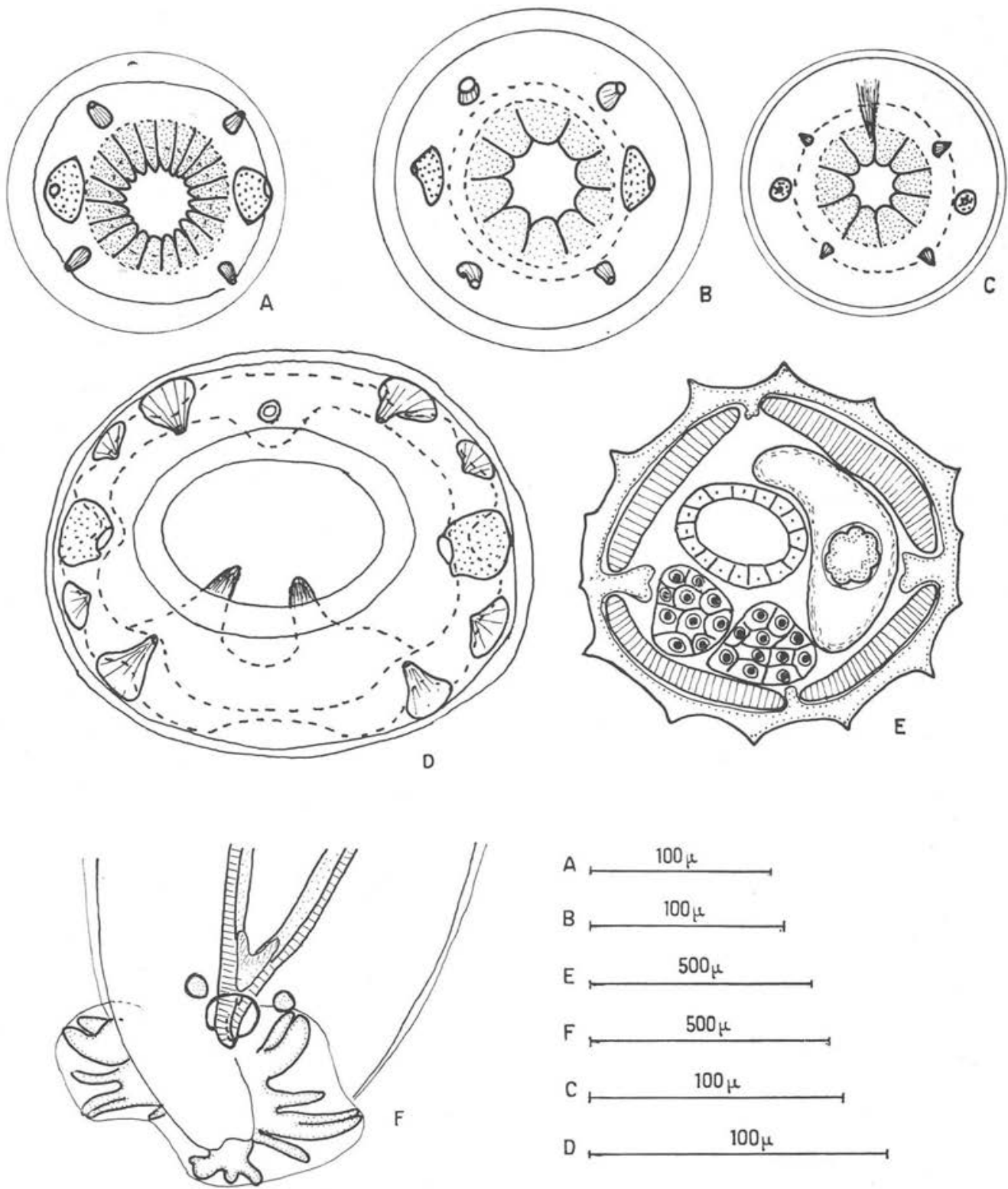

FIG. 9. - Vue apicale de Esophagostomum columbianum. B. Vue apicale de Esophagostomum bifurcum. C. Vue apicale de Esophagostomum longicaudum. D. Vue apicale de Globocephalus urosubulatus. E. Coupe transversale d'une femelle de Cooperic pectinata. F. Extrémité caudale d'un mâle de Stephanurus dentatus 


\section{C. - Compléments iconographiques sur quelques Espèces}

1. Nous donnons (fig. 9) une vue apicale montrant les différences dans le diamètre céphalique et le nombre d'éléments de la corona radiata de Oesophagostomum bifurcum (fig. 9 B), Oe. columbianum (fig. 9 A) et Oe. longicaudum (fig. 9 C).

2. Sur une vue apicale de Globocephalus urobulatus on note la présence de quatre papilles du cycle externe, le conduit de la glande œsophagienne dorsale, les deux dents ventrales, une légère division des amphides et la section irrégulière de la capsule buccale (fig. $9 \mathrm{D}$ ).

3. Un coupe transversale de Cooperia pectinata montre 14 crêtes longitudinales inégalement saillantes (fig. $9 \mathrm{E}$ ).

4. Une vue ventrale de la bourse caudale de Stephanurus dentatus montre l'asymétrie de cette bourse et l'inégalité des côtes des deux lobes latéraux (fig. 9 F).

RÉfÉRENCES Bibliographiques: Amberson (J. M.) ; Schwartz (E.), 1952 ; Berghe (L. van den), 1934 ; Railliet (A.) et Henry (A. C. L.), $1955 a$ et $b$; Sandground (J. H.), 1929 ; Smith (A. J.), Fox (H.) et White (C. Y.), 1908 ; Yamaguti (S.), $1954 b$.

\section{Conclusions}

Les 23 espèces de Strongylidés parasites de Mammifères congolais que nous avons étudiées représentent selon les cas, soit des espèces cosmopolites, soit des espèces purement africaines. Dans chacun de ces deux cas, l'intérêt parasitologique de ces Nématodes est différent selon qu'il s'agit :

$1^{\circ}$ d'espèces simplement parasites d'animaux domestiques (pour les cosmopolites) ou d'animaux sauvages (pour les africaines),

$2^{\circ}$ d'espèces à la fois parasites des uns et des autres et provenant vraisemblablement des animaux domestiques (pour les cosmopolites) ou d'animaux sauvages (pour les africaines).

Dans chacun de ces deux cas, nous rencontrons également une espèce qui, en raison de son parasitisme chez l'homme et chez les Primates, mérite une discussion particulière.

A. Espèces cosmopolites. Elles se répartissent en deux groupes d'intérêt parasitologique différent :

1. Espèces parasitant seulement les animaux domestiques. - Ce groupe d'espèces ne présente guère d'intérêt zoogéographique et il nous suffira d'énumérer les six représentants: Globocephalus urosubulatus, Stephanurus dentatus, Metastrongylus (= Choerostrongylus) pudendotectus, Oesophagostomum longicaudum, Cyathostomum catinatum et Cyathostomum nassatum. 
2. Espèces parasites à la fois d'animaux domestiques et d'animaux sauvages. - Ce groupe comprend huit espèces dont il est difficile de préciser si elles sont des parasites d'animaux sauvages adaptés secondairement à des animaux domestiques ou, inversement des parasites d'animaux domestiques contaminant des animaux sauvages. Toutefois, le cosmopolitisme de ces espèces et le fait que les Strongylidés purement africains d'animaux sauvages ne sont que rarement parasites d'animaux domestiques (cf. B.) laissent à penser que le second cas est le plus fréquent, quel que soit l'ordre chronologique de la découverte respective des hôtes domestiques ou sauvages. $\mathrm{Au}$ surplus, nos Strongylidés d'animaux indigènes proviennent, pour certains, d'individus gardés en captivité au Jardin Zoologique de Brazzaville.

Six de ces espèces ont été simplement retrouvées par nous au Congo chez leurs hôtes domestiques (cf. introduction); ce sont :

- Cyathostomum goldi, et

- Cyathostomum longibursatum parasites cosmopolites d'Equidés domestiques (âne, mulet, cheval) connus d'autre part comme parasites respectivement du zèbre de Chapman (cf. Boulenger, 1920) et de Hippotigris zebra (cf. Mönnig, 1926, 1928) ;

- Oesophagostomum columbianum parasite très cosmopolite du mouton et de la chèvre, signalé en outre chez de nombreux Ruminants sauvages (cf. Mönnig, 1926, 1928) ;

- Oesophagostomum radiatum parasite cosmopolite du bœuf et trouvé aussi chez Bubalis bubalis ;

- Bunostomum phlebotomum parasite cosmopolite du bœuf que l'on a observé également chez de nombreux Ruminants sauvages et notamment chez Adenota kob leucotis de l'ancien Soudan Anglo-Egyptien (cf. Myers, Wolfgang et Kuntz, 1960), et

- Haemonchus contortus parasite courant du mouton, du bœuf et de la chèvre, connu par ailleurs chez des Ruminants sauvages (cf. Le Roux, 1930), entre autres Limnotragus spekei grattus (cf. Berghe, 1943).

Deux de ces espèces par contre ont été observées par nous chez des hôtes sauvages, il s'agit de :

- Gaigeria pachyscelis parasite courant du mouton, de la chèvre et du bœuf en Inde et en Afrique, signalé en Afrique chez Aepyceros melampus (cf. Le Roux, 1930, Mönnig, 1928) que nous avons trouvé chez Syncerus nanus, et

- Trichostrongylus colubriformis déjà signalé chez les Ruminants sauvages (cf. Le Roux, 1930) et des Simiens africains (cf. Railliet et Henry, 1918) que nous avons trouvé chez Ourebia ourebi dorcas.

3. Ternidens deminutus, parasite de l'Homme et des Simiens. - Ce parasite, connu seulement de l'Ancien Monde, semble répandu dans toute l'Afrique et l'Asie. Il a été décrit des genres Pan (cf. Schmid, 1937), en Haute-Guinée portugaise, Gorilla au Congo de Brazzaville (cf. Mithchell, 1906), Cercopithecus en Rhodésie du Sud et au Congo ex-Belge (cf. Gedoelst, 1915, Blackie, 1932, Amberson et Schwartz, 1952), Mandrillus en Rhodésie du Sud (cf. Blackie, 1932), Zati au Tonkin et en 
Inde (cf. Noc, 1908, Brumpt, 1910, Houdemer, 1927), Macacus aux Célèbes, en Indochine et à Sumatra (cf. Mac Clure, 1933, Houtdemer, 1938), Cercocebus au Congo ex-Belge (cf. Vuylstèke, 1956). Nous l'avons rencontré chez Cercopithecus cephus du Congo de Brazzaville et proposons la mise en synonymie d'avec Ternidens simiae Yamaguti, 1954 décrit de Simiens sp. des Célèbes.

B. Espèces africaines. Situé dans la sous-région occidentale de la région zoogéographique éthiopienne, encerclé par les provinces de l'Est et du Sud africain, du Nord-Est africain et soudanaise, le bloc forestier congolais présente une faune mammalienne de type ubiquiste (forestier, soudanien, sahelo-soudano-guinéen et austral) (Dekeyser, 1955). En ces conditions l'on devrait s'attendre à y trouver une faune d'helminthes indigènes composée d'espèces déjà connues dans ces différentes provinces ou étroitement apparentées. C'est ce que nous a montré notre étude qui permet ainsi de souligner l'uniformité de la faune des Strongylidés de la région éthiopienne, et ce que nous précisons à propos de chacune des espèces considérées.

\section{Parasites d'animaux sauvages.}

Ce sont :

- Agriostomum gorgonis var. congolensis nov. Cette variété parasite de Kobus defassa présente d'étroites affinités avec Agriostomum gorgonis Le Roux décrit de Gorgon taurinus et Strepsiceros strepticeros en Afrique du Sud (cf. Le Roux, 1929).

- Ostertagia sissikoi n. sp., parasite de Sylvicapra grimmia est voisine de Ostertagia harrisi Le Roux décrit chez Tragelaphus sylvaticus en Afrique du Sud (cf. Le Roux, 1930).

- Cooperia fülleborni, parasite de Sylvicapra grimmia, a été décrit en Afrique du Sud chez Kobus ellipsiprymus et Damaliscus lunatus (cf. Hung, 1926).

- Cooperia chabaudi n. sp., parasite de Cephalophus dorsalis présente des affinités avec Cooperia minor du Congo ex-Belge (cf. de Brito Guterres, 1947), Cooperia yoshidai décrit chez Redunca arundinum en Afrique du Sud (Mönnig, 1939 et Cooperia ershovi du bœuf en Chine (Wu, 1958).

- Ashworthius lerouxi n. sp. parasite de Syncerus nanus nanus ressemble à Ashworthius pattoni de Tragelaphus sylvaticus en Afrique du Sud (Le Roux, 1930) et Ashworthius martinagliai de Boselaphus tragocamelus en Inde (Ortlepp, 1925).

\section{Parasites à la fois d'animaux sauvages et domestiques.}

Nous pensons pouvoir ranger ici :

- Cooperia pectinata surtout parasite de Ruminants sauvages et que nous avons retrouvé chez le bœuf, comme en Afrique du Sud (Mönnig, 1928, Le Roux, 1930). Cette espèce a été probablement introduite à Madagascar, puisque nous en avons examiné des exemplaires provenant d'un bœuf abattu à Tananarive ;

- Oesophagostomum rousseloti n. sp. que nous décrivons chez un porc du Jardin Zoologique de Brazzaville, mais qui se rapproche plus particulièrement d'espèces décrites chez les Suidés sauvages d'Afrique. 


\section{Oesophagostomum bifurcum, parasite de l'Homme et des Primates.}

Quel que soit le nom donné sans commentaires suffisants par Houdemer (1927 et 1938) à des parasites de Macacus nemestrinus et Macacus cynomolgus d'Asie, nous pensons avec Travassos et Vogelsang (1932) que cette espèce est purement africaine. Connue de l'Homme (Brumpt, 1902, Joyeux, 1933) et des Primates, elle a été signalée chez Mandrillus leucophaeus (Railliet et Henry, 1910), Cercocebus sp. (Lavier et Le Roux, 1928, Neveu-Lemaire, 1935) chez des Simiens indéterminés (Travassos et Vogelsang, 1932), Papio anubis (Berghe, 1943) et Cercopithecus aethiops (Vuylsteke, 1956) au Congo ex-Belge. Nous l'avons retrouvé chez Mandrillus leucophaeus de la région de Brazzaville.

\section{Résumé}

Nous avons étudié 23 espèces de Strongylidés comprenant quatre espèces et une variété nouvelles.

Classées d'après leurs hôtes, les 19 espèces déjà connues que nous avons rencontrées sont :

- chez Equus caballus : Cyathostomum catinatum, Cyathostomum longibursatum, Cyathostomum nassatum, Cyathostomum goldi;

- chez Sus scrofa domestica: Globocephalus urosubulatus, Stephanurus dentatus, Oesophagostomum longicaudum, Metastrongylus (Choerostrongylus) pudendotectus;

- chez Cercopithecus cephus et Simiens sp.: Ternidens deminutus;

- chez Mandrillus leucophaeus: Oesophagostomum bifurcum;

- chez Bos taurus et Bibos indicus: Bunostomum phlebotomum, Oesophagostomum radiatum, Cooperia pectinata;

- chez Ovis aries: Oesophagostomum columbianum, Trichostrongylus colubriformis, Haemonchus contortus;

- chez Syncerus nanus nanus: Gaigeria pachyscelis ;

- chez Ourebia ourebi dorcas: Trichostrongylus colubriformis, et

- chez Sylvicapra grimmia: Cooperia fülleborni.

Parmi ces espèces, certaines (Oesophagostomum columbianum, Oe. longicaudum, Cooperia fülleborni) donnent lieu à quelques compléments descriptifs ; de plus nous redécrivons Ternidens deminutus Railliet et Henry, 1905 et nous proposons la mise en synonymie de $T$. simiae Yamaguti, 1954.

Les unités taxonomiques nouvelles que nous décrivons sont:

- Oesophagostomum rousseloti n. sp., parasite de Sus scrofa domestica, proche de Oe. watanabei Yamaguti, 1961, mais s'en différenciant par une côte postéro-latérale sans rameau accessoire à sa base et surtout par ses spicules longs de 2,3 $\mathrm{mm}$; nous pensons qu'il s'agit d'un parasite de Suidés sauvages égaré chez le porc ; 
- Ostertagia sissikoi n. sp. trouvé chez Sylvicapra grimmia, proche de $O$. harrisi Le Roux, 1930, mais s'en différenciant par ses spicules de 0,250 mm, l'absence de gubernaculum et un utérus postérieur non atrophié ;

- Cooperia chabaudi n. sp. de Cephalophus dorsalis, proche de C. minor de Brito Guterres, 1947, mais s'en différenciant par un mâle plus grand, un ovéjecteur beaucoup plus long et une côte dorsale non digitée distalement et sans rameaux accessoires à sa bifurcation proximale ;

- Ashworthius lerouxi n. sp. chez Syncerus nanus, proche de A. pattoni Le Roux, 1930, mais s'en différenciant par une côte postéro-latérale plus courte, des spicules de structure différente et la position strictement antérieure de l'ouverture buccale, et

- Agriostomum gorgonis var. congolensis nov. chez Kobus defassa, très voisine d'A. gorgonis Le Roux, 1929, mais dont nous faisons toutefois, une variété à cause de ses spicules plus longs, de sa côte externo-dorsale plus courte et de son plateau coupant presque inexistant.

L'étude, pour chaque espèce, de ses hôtes et de sa répartition nous permet, en conclusions de distinguer, dans la faune des Strongylidés du Congo, des parasites cosmopolites d'animaux domestiques dont plusieurs ont contaminé des animaux sauvages et des parasites africains d'animaux sauvages dont certains ont été retrouvés chez des animaux domestiques.

Nous soulignons, en ce qui concerne ces éléments purement africains, l'uniformité de la faune des Strongylidés de Mammifères de la zone éthiopienne.

\section{Bibliographie}

Alicata (J. E.), 1935. - Oesophagostomum longicaudum Goodey, 1925, a synonym of Oesophagostomum quadrispinulatum (Marcon, 1901). J. Parasitol., XXI (3), 215-216.

Altaev (A.-K.), 1953. - [Deux nouveaux strongylides du mouton au Daghestan] (en russe). Trav. d'Helminthol. pour le $75^{\circ}$ anniver. de K. I. Skrjabin, 17-24.

Amberson (J. M.) et Schwartz (E.), 1952. - Ternidens deminutus Railliet et Henry, a nematode parasite of Man and Primates. Ann. Trop. Med. and Parasitol., XLVI (3), 227-237.

ANDREEVA (N.-K.), 1958. - [Atlas des Helminthes (Strongylata) des ruminants domestiques et sauvages au Kazakhstan] (en russe); 215 pp., Illust., Inst. Vétér. de la filiale du Kazakht. de l'Académie Lénine d'Agriculture.

ANTIPIN (D. N.), 1931. - Cooperia surnabada nov. sp. aus dem Dünndarm des Rindes Aserbaidjans. Deutsch. Tierärztl. Wchnschr., XXXIX (30), 469-471, fig. 1-6.

Asadov (S.-M.), 1954. - [Contribution à l'étude de la faune helminthologique de Capreolus capreolus L. en Azerbaidzhan] (en russe). Izvest. Akad. Nauk Azerbaidzhan SSR, IX, 33-45.

BAER (J.-G.), 1950. - Etude critique des Helminthes de l'Okapi. Acta Trop. Bâle, VII (2), 164-186. 
Baylis (H. A.), 1920. - Notes on some parasitic Nematodes from East Africa. Ann. and Mag. Nat. Hist., sér. 9, VI, 283-295.

- - 1923. - Report on the collection of parasitic Nematodes mainly from Egypt. Parasitol., XV, 1 et 14-24.

- - 1929. - Some parasitic Nematodes from the Uluguru and Usambara Mountains, Tanganyika Territory. Ann. and Mag. Nat. Hist., sér. 10, IV, 372-381.

—_, 1930. - Mission saharienne Auguiéras-Draper 1927-1928. Parasitic Nematodes. Bull. Mus. Hist. Nat. Paris, II (2), 117-130.

- - 1936. - Some parasitic worms from British Cameroons. Ann. and Mag. Nat. Hist., sér. 10, XVII, 257-272.

- - 1938 a. - A new species of Cooperia (Nematoda) from cattle and sheep. Ann. and Mag. Nat. Hist., sér. 11, I (1), 68-73.

- - 1938 b. - Notes on some species of the Nematode genus Conperia from cattle and sheep. Vet. Rec., L (10), 283-285.

- - 1939. - Records of some parasitic worms from the Belgian Congo. Ann. and Mag. Nat. Hist., sér. 11, III (18), 625-629.

- - 1940. - On a further collection of parasitic worms from the Belgian Congo. Ann. and Mag. Nat. Hist., sér. 11, V (29), 401-416.

- and Daubney (R.), 1922. - Report on the parasitic Nematodes in the collection of the zoological survey of India. Mem. Ind. Mus., VII, 263-347, fig. 1-75.

Berghe (L. VAN DEN), 1934. - L'existence de Ternidens deminutus au Katanga. Ann. Soc. Belge de Med. Trop., XIV (2), 189-191.

—, 1937. - Contribution à l'étude des parasites de l'Okapi. Rev. Zool. et Botan. Africaines, XXIX (2), 141-150, XXX (1), 117-139.

-, 1943. - Enquête parasitologique. II. Helminthes parasites. Fasc. 2, Mission van Den Berghe (1936). Explor. Parc Nat. Albert, 3-30, 12 pl.

BLACKIE (W. K.), 1932. - A helminthological survey of Southern Rhedesia. Lond. Sch. Hyg. Trop. Med., Mem. sér. 5, 91 pp.

Boulenger (Ch. L.), 1917. - Sclerostomes parasites of the horse in England. II. New species of the genus Cylichnostomum. Parasitol., IX (2), 203-231.

-, 1920 a. - Sclerostomes of the donkey in Zanzibar and East Africa. Parasitol., XII (1), 30-32.

—, 1920 b. - On some Nematodes parasites of the zebra. Parasitol., XII (2), 98-107.

-, 1921. - Strongylid parasites of horses in the Penjab. Parasitol., XIII (4), 321-325.

-, 1923. - A collection of Nematode parasites from Zanzibar. Parasitol., XV (2), 113-121.

-, 1926. - Report on a collection of parasitic Nematodes, mainly from Egypt. Part. IV Trichostrongylidae and Strongylidae. Parasitol., XVIII (1), 86-100.

Brito-GutTerRes (J. DE), 1946. - Un nouveau Nématode parasite du duodénum du mouton. Rev. Med. Vet., Lisbonne, XLI (319), 431-435.

—, 1947. - Contribuição para o estudo das verminoses gastro-intestiı ais dos bovinos africanos. A tricoestrongilinose bovina em Africa. Diss. (Dr. Med. Vet.), Lisboa, 92 pp., Illust. 
—, 1949. - Haemonchus santomei n. sp. parasite de la caillette du bœuf de San Tomé. Ann. Parasitol., XXIV (1-2), 93-97.

-, 1952. - Contribuição ao estudo da helmintologia veterinária africana. Ann. Inst. Med. Trop. Lisboa, IX (4), 1301-1335.

Cameron (T. W. M.), 1939. - Animal parasites of wild animals. 13. Internat. Vet. Cong. (Zürich), 13-22.

Chabaud (A.-G.), 1959. - Remarques sur la systématique des Nématodes Trichostrongyloïdea. Bull. Soc. Zool. France, LXXXIV (5-6), 473-483.

- (sous presse). - Nématodes parasites de Vertébrés. Traité de Zool., IV (2), Masson et $C^{10}$ éditeurs, Paris.

Chapin (E. A.), 1925. - New Nematodes from North American mammals. J. Agric. Research U.S. Dept. Agric., XXX (7), 677-681, fig. 1-4.

Clure (G. W. MAC), 1933. - Nematodes parasites of mammals from specimens collected in the New York Park, 1931. Zoologica, N. Y., XV, 29.

Cram (E. B.), 1925. - Cooperia bisonis a new Nematode from the buffalo. J. Agric. Research, U.S. Dept. Agric., XXX (6), 571-573, fig. 1-7.

Curson (H. H.), 1928. - Metazoan parasites from Zululand. South. Afric. J. Nat. Hist. Pretoria, VI, 181-187.

DAUBNEY (R.), 1924. - Description of a new Nematode Oesophagostomum mwanzae, from the warthog. Ann. and Mag. Nat. Hist., sér. 9, XIII, 542-546, fig. 1-4.

-, 1926. - Oesophagostomes from the wart hog. Ann. and Mag. Nat. Hist., sér. 9, XVII, 11-17, fig. 1-2.

-, 1932. - Oesophagostomum multifoliatum n. sp. an undescribed Nematode from sheep and goats. Parasitol., XXIV (2), 265-267, fig. 1-5.

DeKeyser (P.-L.), 1955. - Les Mammifères de l'Afrique Noire française. Initiations africaines, $I$ ( $2^{\circ}$ édition), I.F.A.N., Dakar.

Diaz-Ungria (C.), 1954. - Dos species de estrongilos, parasitos del caballo, nuevas para Venezuela. Mem. de la Soc. de Cienc. Nat., La Salle; XIV (39), 261-271.

Dikmans (G.), 1931. - Two new species of Nematode worms of the genus Ostertagia from the virginia deer with a note on Ostertagia Lyrata. Proc. U.S. Nat. Mus., LXXIX (6), 1-6, pl. 1-2.

Duthy (B. L.), 1948. - Three new Nematodes of the genus Oesophagostomum from the East African warthog, Phacochoerus aethiopicus. Ann. and Mag. Nat. Hist., sér. 11, XIV, 280-288.

Gedoelst (L.), 1916. - Notes sur la faune parasitaire du Congo Belge. Rev. Zool. Africaine, $\mathrm{V}$ (1), 1-90.

-, 1924. - Notes de Parasitologie congolaise. Ann. Soc. Belg. Med. Trop., IV (2), 1-7.

GendRe (E.), 1909 a. - Notes d'Helminthologie africaine (1 $1^{\text {re }}$ note). Proc. Verb. Soc. Linn. Bordeaux, LXIII, 29-33, fig. 1-6.

—, 1909 b. - Notes d'Helminthologie africaine (2e note). Ibid., LXIII, 33-41.

—, 1909 c. - Notes d'Helminthologie africaine ( $3^{\circ}$ note). Ibid., LXIII, 74-81.

-, 1913. - Notes d'Helminthologie africaine (4e note). Ibid., LXVII, 106-112, fig. 1-6.

—, 1921 a. — Sur deux espèces de Nématodes africains. Ibid., LXXIII, 28-36. 
—, 1921 b. - Notes d'Helminthologie africaine ( $5^{\circ}$ note). Ibid., LXXIII, 49-55, fig. 1-10.

—, 1921 c. - Notes d'Helminthologie africaine (6 ${ }^{\circ}$ note). Ibid., LXXIII, 148-156, 11 fig.

Goodey (T.), 1924. - Some new members of the genus Oesophagostomum from the roan antelope and the wart hog. J. Helminth., II (3), 135-148, fig. 1-26.

—, 1925. - Oesophagostomum longicaudum n. sp. from the pig in New Guinea. Ibid., III (1), 45-50, fig. 1-2.

GoRdon (H. Mc. L.), 1932. - Some Helminth parasites reported from Australia for the first time, with a description of Cooperia mc masteri, sp. nov. from a calf. Austrnl. Vet. J., VIII (1), 2-12, fig. 1-6.

Grigorian (G. A.), 1951. - [Etude des Helminthes parasites des Ruminants sauvages en Roumanie et leur rôle dans la transmission des helminthiases chez les petits Ruminants domestiques] (en russe). Trudy Gelmint. Lab., Akad. Nauk SSR, V, 308-310.

—, 1952. - [Contribution à la connaissance des Helminthes parasites de Capreolus capreolus en Arménie russel (en russe). Izvest. Akad. Nauk Armiansk. SSR, V (4), 57-65.

Henry (A.) et Joyeux (Ch.), 1930. - Contribution à la faune helminthologique de la HauteGuinée française. Bull. Soc. Path. Exot., XIII, 176-182.

Houdemer (F.-E.), 1927. - Observations et recherches sur lhygiène et la pathologie animale du Tonkin. Thèse Doct. Vet. Paris, 240 pp.

—, 1938. - Recherches de Parasitologie comparée indochinoise. Libr. Le François, Paris.

HsiUng (T. S.) et Kung, 1955. - A preliminary report on the investigation of the oesophagostomes of the domestic animals of China with the description of a new species. Scienc. Rep. Peking. Agric. Univ., I (1), 147-170.

HUNG (See Lu), 1926. - Ueber Zwei neue parasitische Nematoden, Cooperia fuelleborni, aus dem Wasserbock und Oesophagostomum maurum aus dem Mohren makak. Arch. Schiffs-u.-Tropen-Hyg., XXX (9), 421-430, fig. 1-13.

IHLE (J. E. W.), 1921. - On the genus Cylicostomum. Ann. Trop. Med. and Parasitol. XV (4), 397-402.

Joyeux (Ch.), 1923. - Recherches sur la faune helminthologique africaine. Arch. Inst. Pas. teur, Tunis, XII, 119-167 et 328-338.

LANe (C.), 1923. - Some Strongylata. Parasitol., XV (4), 348-364, 80 fig.

LÉGer (M.) et BÉdier (E.), 1923. - Le parasitisme intestinal en A.O.F. Bull. Com. Et. Hist. et Scient. de l'A.O.F., 1-30.

LEIPER (R. T.), 1908. - The occurence of a rare sclerostome of man in Nyasaland. J. Trop. Med. and Hyg., XI (12), 181-184, fig. 1-4.

-, 1911 a. - Notes of recent and some new records of Helminths in man of which there are few records. J. London School. Trop. Med., 1 (1), 16-19.

—, 1911 b. - Some new parasitic Nematodes from tropical Africa. Proc. Zool. Soc. London (2), 549-555, fig. 140-144.

-, 1911 c. - Check. list of Helminthes parasitic in Equines. J. London Sch. Trop. Med., 1, (1), 22-26.

-, 1936. - Report on the Helminth parasites of the okapi recently living in the Society's gardens. Proc. Zool. Soc. London, part. 4, 949. 
LE Roux (P.-L.), 1924. - Helminths collected from equines in Edinburgh and London. J. of Helminth., II (3), 111-134.

—, 1929 a. - On a hookworm (Agriostomum gorgonis, sp. nov.) from the blue wildebeest (Gorgon taurinus) in the Transvaal. 15. Ann. Rep. Director Vet. Serv., Dept. Agric. Union South Africa, I, 481-491, 13 fig.

-, 1929 b. - A preliminary report on three new members of the genus Haemonchus, Cobb, 1898, from antelopes in South Africa. 15. Ann. Rep. Director Vet. Serv., Dept. Agric. Union South Africa, I, 451-463, fig. 1-24.

-, 1929 c. - Two species of Haemonchus, Cobb, 1898, parasiting the camel in the Cape Province. 15. Ann. Rep. Director Vet. Serv., Dept. Agric. Union South Africa, I, 439-450, fig. 1-15.

-, 1930 a. - Helminthiasis of Domestic Stock in the Union of South Africa. J. South. Africa Vet. Med, Ass., I (4), 1-23.

-, $1930 \mathrm{~b}$. - On two new Helminths from the abomasum of the bushbuck in Zululand, Natal. 16. Rep. Director Vet. Serv., Dept. Agric. Union South Africa, 233-241, fig. 1-13.

-, 1936. - On Schwartziella, a new Nematode genus for Cooperia nodulosa Schwartz, 1928. J. Helminth., XIV (2), 113-118 et 1-6, fig. 1-3.

-, 1940. - On the division of the genus Oesophagostomum Molin, 1861, into subgenera and the creation of a new genus for the Oesophagostomes of the wart hog. J. Helminth., XVIII (1), 1-20.

—, 1950. - A trichostrongylid, Paracooperia mazabukae sp. nov., from a wild Ruminant, the oribi, in northern Rhodésia. J. Helminth., XIV (1-2), 28-32.

LING (M. T.), 1959. - On new species of Oesophagostomum (Nematoda, Trichonematidae) from pigs in Chekiang. Acta. Zool. Sinica, XI, (1), 24-27.

Lizcano-Herrera (J.), 1951. - Estrongilosis intestinales equinas. Rev. Iber. de Parasitol., XI, (3), 283-306.

-, 1958. - Contribución al conocimiento de los Oesophagostomum del Cerdo. Oesophagostomum dentatum (Rudolphi 1803), Oe. granatensis nov. specie, y Oe. longicaudum (Goodey 1925), nuevo en Europa. Rev. Iber. Parasitol., XVIII (3), 221-226 et 231.

Looss (A.), 1901. - The Sclerostomidae of horses and donkeys in Egypt. Rec. of Egypt. Gov. School of Med., Cairo.

MAPlestone (P.-A.), 1930. - Nematode parasites of pigs in Bengal. Kec. Indian Mus., XXXII (2), 77-105, fig. 1-37.

-, 1931. - Oesophagostomum conicum. Ann. Trop. Med. and Parasitol., XXV (3-4), 551.

MöNNIG (H. O.), 1923. - On some new South African parasitic Nematodes. Tr. Roy. Soc. South Africa, XI (2), 105-117, fig. 1-12.

-, 1924. - South African parasitic Nematodes. 9-10. Rep. Director Vet. Educ. and Research, Dept. Agric. Union South Africa, 435-478, fig. 1-46.

-, 1925. - A new Trichostrongylus from South African sheep. Tr. Roy. Soc. South Africa, XII (4), 243-247, fig. 1-3.

—, 1926 a. - Three new Helminths. Tr. Roy. Soc. South Africa, XIII (3), 291-298, fig. 1-12.

—, 1926 b. - Helminthological notes 11-12. Rep. Director Vet. Serv., Dept. Agric., South Africa, 221-228. 
-, 1928. - Chek list of the worm Parasites of Domesticated Animals in South Africa 13-14. Rep. Director Vet. Educ. and Research., Dept. Agric. Union South Africa, 801-837.

-, 1929 a. - Filarinema flagrifer n. gen., s. sp., a trichostrongylid parasite of the Kangaroo. 15. Ann. Rep. Director Vet. Serv., Dept. Agric. Union South Africa, I, $307-$ 310, fig. 1-5.

—, 1929 b. - Agriostomum equidentatum n. sp., a hookworm of the springbuck 15. Ann. Rep. Director. Vet. Serv., Dept. Agric. Union South Africa, I, 311-316, 8 fig.

—, 1931 a. - On a new hookworm, Ancylostoma martinagliai, from a jackal. J. South African Vet. Med. Ass. II (2), 125-127, fig. 1-6.

-, $1931 \mathrm{~b}$. - Wild antelopes as carriers of Nematode parasites of Domestic Ruminants Part. I, 17. Rep. Dir. Vet. Serv. and Anim. Ind., Union South Africa, 233-254.

-, 1932 a. - The genus Agriostomum with a description of A. cursoni n. sp. J. South African Vet. Med. Ass. III (1), 16-21, fig. 1-6.

-, 1932 b. - New strongylid Nematodes of antelopes (preliminary notes). J. South African Vet. Med. Ass., III (4), 171-175.

-, 1932 c. - Wild antelopes as carriers of Nematode parasites of Domestics Ruminants. Part. II, 18. Rep. Director Vet. Serv. and Anim. Indust., Union South Africu, 153-172.

-, 1939. - Cooperia yoshidai n. sp., a Nematode parasite of the reedbuck, Redunca arundinum. Vol. Jub. Yoshida, II, 291-294.

MYers (B. J.), Wolfgang (R. W.) et KuntZ (R. E.), 1960. - Helminth parasites from Vertebrates taken in the Sudan (East Africa). Can. J. Zool., XXXVIII, 834-836.

-, 1962. - Helminth parasites of Reptiles, Birds and Mammals in Egypt. VII. Chek-list of Nematodes collected from 1948 to 1955. Can. J. Zool., LX, 532-538.

Neveu-Lemaire (M.), 1927. - Les Oesophagostomes des phacochères. Ann. Parasitol., V (3), 214-219.

—, 1928. - Essai de Mammalogie médicale. II. Les Mammifères hôtes intermédiaires ou hôtes définitifs des Helminthes parasites de l'homme et ceux qui hébergent des parasites qui leur sont communs avec l'espèce humaine. Ann. Parasitol., VI (1), 356-380.

—, 1935. - Un nouvel hôte d'Oesophagostomum (Conoweberia) bifurcum (Creplin, 1849). Ann. Parasitol., XIII (3), 203-206.

—, 1936. — Traité d'Helminthologie médicale et vétérinaire. 1.514 pp., 787 fig., Vigot Frères, Paris.

Orlov (I. V.), Belova et Gnedina (M. P.), 1931. - Ein neuer Nematode Ostertagia dahurica nov. sp. aus dem Labmagen des Schafes. Tierärztl. Rundschau, XXXVII (41), 723-725, fig. 1-5.

——, 1933. - Sur la reconstruction de la systématique du genre Ostertagia Ransom, 1907. Ann. Parasitol., XI (2), 96-114, fig. 1-14.

ORTLEPP (R. J.), 1935. - On some Helminths from the \&nylgiae» Boselaphus tragocamelus (Pall.) with observations on the parasitic larval stages of the stomache worm Ashworthius martinagliai sp. n. Onderstepoort J. Vet. Sc. and Animal Indust., V (1), 43-50, fig. 1-9. 
Popova (Z. G.), 1937. - [Un nouveau Nématode du bétail, Ostertagia kolchida n. sp.] (en russe). Papers on Helminthol. publish. in commemor. of the 30 years activities of K. I. Skrjabin, 1937, Moscou, 497-499.

—, 1958. - [Strongylidés des animaux et de l'homme. Trichonematidae] (en russe). Osnovy nematodologii, VII, Moscou.

Pushmenkov (E. P.), 1937. - [Trichostrongylides de la caillette des Rennes] (en russe). 550-559. Papers on Helminthol. publish. in commemor. of the 30 years activities of K. J. Skrjabin, 1937, Moscou.

Railliet (A.) et Henry (A.-C.-L.), 1905 a. - Le Triodontophorus deminutus, nouveau Sclérotomien parasite de l'homme, et la cachexie africaine. Bull. Mus. Hist. Nat., Paris, XI (4), 269-272.

- - 1905 b. - Un nouveau Sclérotomien (Triodontophorus deminutus nov. sp.), parasite de l'homme. C.R. Soc. Biol., LVIII (13), 569-571.

- - $1909 a$. — Sur la classification des Strongylidae. 1-Metastrongylinae. C.R. Soc. Biol., Paris, LXVI (2), 85-88.

- - 1909 b. - Sur la classification des Strongylidae. 2-Ankylostominae. C.R. Soc. Biol., Paris, LXVI (4), 168-171.

- - 1910. - Quelques Helminthes nouveaux peu connus du groupe des Bunostomiens. Bull. Soc. Path. Exot., III (5), 311-315.

- - 1912. - Les Oesophagostomes parasites de l'homme. Arch. Parasitol., XIV (4), 562583.

- - 1913 a. - Un nouveau Strongylidé des Singes. Bull. Soc. Path. Exot., VI, 264-267.

- - 1913 b. - Sur les Oesophagostomiens des Ruminants. Bull. Soc. Path. Exot., IV (7), 506-511, fig. 1-4.

- -, 1918. - Nématodes parasites du Congo Belge. Bull. Soc. Path. Exot., XI (2), 82-98.

- - et Bauche (J.), 1919. - Un nouveau Strongylidé du Porc. Bull. Soc. Path. Exot., XII (6), 324-332, fig. 1-5.

Ransom (B. H.), 1907. - Notes on parasitic Nematodes, including descriptions of new genera and species, and observations on life histories. Circular (116), Bureau Animal Indust., U.S. Dept. Agric., 7 pp.

-, $1911 a$. - The Nematodes parasitic in the alimentary tract of cattle, sheep and other Ruminants, Bull. (127), Bureau Animal Indust., U.S. Dept. Agric., 132 pp., fig. 1-152.

—, 1911 b. - Two new species of parasitic Nematodes. Proc. U.S. Nat. Mus., XLI, 363-369, fig. 1-7.

SANDGround (J. H.), 1928. - Some new Cestode and Nematode parasites from Tanganyika Territory. Proc. Boston Soc. Nat. Hist., XXXIX (4), 131-150, pl. XVI-XXI.

-, 1929. - Ternidens deminutus (Railliet et Henry) as a parasite of man in southern Rhodesia, together with observations and experimental infection studies on an unidentified Nematode parasite of man from this region. Ann. Trop. Med. and Parasitol., XXIII (1), 23-32, fig. 1, pl. 2.

-, 1930. - Notes and descriptions of some parasitic Helminths collected by the expedition. Contrib. Dept. Med. and Inst. Trop. Biol. and Med., Harward Univ., I (5), 462-486. 
—, 1931. - Studies on the life history of Ternidens deminutus, a Nematode parasite of man, with observations on its incidence in certain regions of southern Africa. Ann. Trop. Med and Parasitol., XXV (2), 147-184, pl. 5-6.

—, $1933 a$. - Report on the Nematode parasites collected by the Kelley-Roosevelt's expédition to Indo-China with descriptions of several new species. Part. 1. Parasites of Birds. Part 2. Parasites of Mammals. Ztschr. Parasitenk., Berlin, V (3-4), 542-583.

-, $1933 \mathrm{~b}$. - Report on the scientific results of an expedition to the southwestern highlands of Tanganyika Territory. VI. Parasitic Nematodes from East Africa and Southern Rhodesia. Bull. Mus. Comp. Zool. Harward Coll., LXXV (6), 263-293, fig. 1-14.

-, 1933 c. - Two new Helminths from Rhinoceros sondaicus. J. Parasitol., XIX (3), 192204, fig. 1-6.

—, 1937. - Three new parasitic Nematodes from the Belgian Congo. Rev. Zool. et Botan. Africaines, XXIX (3), 230-236.

SANKIN (S. L.), 1930. - Ueber eine Nematode beim Schafe (Ostertagia orloffi nov. sp.). Zentralbl. Bakteriol., 1, Abt., Orig. CXVII (7-8), 486-489, fig. 1-3.

Schulz (R. E. S.) (= SHul'Ts), 1933. - Asworthius sidemi n. sp. (Nematoda, Trichostrongylidae) aus einem Hirsch (Pseudaxis hortulorum) des Fernen Ostens. Ztschr. Parasitenk., Berlin V (3-4), 735-739, 8 fig.

Schuurmans-Stekhoven (J. H.), 1937. - Parasitic Nematoda. Expl. Parc. Nat. Albert, Mission G.F. de Witte (1933-1935), Fasc. 4. Bruxelles.

-, 1943. - Parasitic Nematodes from the Belgian Congo. Bull. Mus. Roy. Hist. Nat Bruxelles, XIX, 19.

Schwartz (B.) et Alicata (J. E.), 1930 a. - Species of the Nematode genus Strongyloides parasitic in domestic swine. J. Agric. Research, U.S. Dept. Agric., XL (1), 11-23, fig. 1-12.

- - $1930 \mathrm{~b}$. - Two new species of nodular worms (Oesophagostomum) parasitic in the intestine of domestic swine. J. Agric. Research, U.S. Dept. Agric., XL (6), 517-522, fig. 1-12.

SHIPLeY (A.), 1903. - On a collection of Parasites from the Soudan. Arch. Parasitol., VI (4), 604.

SkadniK (J.), 1935. - Sur la synonymie de quelques espèces de la sous-famille des Trichoneminae. Ann. Parasitol., XIII (4), 307-311.

SKrJabin (K. I.) et Shul'Ts (R. E. S.), 1931. - [Helminthoses humaines (éléments d'helminthologie médicale) à l'usage des médecins, vétérinaires, biologistes et étudiants] (en russe). Part. II, 768 pp., fig. 1-154, pl. 1-4, Moscou.

Skrjabin (K. I.), Shikhobalova (N. P.) et Shul'ts (R. E. S.), 1954. - [Trichostrongyloidea des animaux et de l'homme] (en russe). Osnovy nematodologii, III, 683 pp., Moscou.

Smith (A. J.), Fox (H.) et WhITE (C. Y.), 1908. - Contribution to systematic helminthology. Univ. Penn. Med. Bull., XX (12), 283-294, pl. 2-10.

Stiles (C. W.), Hassal (A.) et Nolan (Mabelle Orleman), 1929. - Key catalogue of parasites reported for Primates (Monkeys and Lemurs). With their possible public health importance. Key catalogue of Primates for which parasites are reported. U.S. Treasury Dept. Health Service, Hyg. Lab., Bull. 152, 409-601.

Ann. de Parasitologie humaine et comparée (Paris), t. 39, 1964, $\mathrm{n}^{\circ} 3$ 
TAYLOR (E. L.), 1924 - Notes on some Nematodes in the Museum of the Liverpool School of Tropical Medicine. Ann. Trop. Med. Parasit., XVIII, 57.

TheILER (G.), 1923. - The Strongylids and other Nematodes parasitic in the intestinal tract of South African equines. 9-10. Thèse (Neuchâtel), 175 pp. LV pl.

Thornton (H.), 1924. - A review of the Oesophagostomes in the collection of Liverpool school of tropical Medicine. Ann. trop. Med. and Parasitol., XVIII (3), 393-408, fig. 1-3.

Travassos (L. P.), 1921. - Contribuiçoes para o conhecimento da fauna helminthologica brasileira. XIII. Ensaio monografico da familia Trichostrongylidae Leiper, 1909. Mem. Inst. Oswaldo Cruz, XIII (1), pp. 1-135, pl. 1-56, fig. 1-250. Traduction française, pp. 1-82.

Travassos (L. P.) et Vogelsang (E. G.), 1932. - Pesquizas helminthologicas realisadas em Hamburgo. X. Contribuição ao conhecimento das species de Oesophagostomum dos Primatas. Mem. Inst. Oswaldo Cruz, XXVI (3), 251-328, pl. 55-91, fig. 1-198.

- -, 1937. — Revisão da familia Trichostrongylidae Leiper, 1912. Monogr. Inst. Oswaldo Cruz (1), 512 pp., 295 pl.

VuYlsteke (C.), 1935. - Nématodes parasites de l'okapi. Rev. Zool. et Botan. Africaines, XXVII (3-4), 313-318.

—, 1956. - Note sur quelques Nématodes parasites avec description de neuf espèces nouvelles. Rev. Zool. et Botan. Africaines, LIII (3-4), 441-477.

Wu SHu Ching, 1958. - On the occurence of Cooperia in the pancreas of chineese cattle, with description of a new species, Cooperia ershovi sp. nov. (Nematoda, Trichostrongylidae). Acta Zool. Sinica, X (1), 19-26.

YAMAgUtI (S.), 1943. - Studies on the helminth fauna of Japan. Part. 43. Mammalian Nematodes IV. Jap. J. Zool., X (3), 427-454.

-, 1954 a. - Studies on the helminth fauna of Japan. Part. 51. Mammalian Nematodes. V. Acta Med. Okayama, IX (1), 105-121.

—, 1954 b. - Parasitic worms mainly from Celebes. Part. 10. Nematodes of Birds and Mammals. Acta Med. Okayama, IX (1), 134-149.

-, 1961 a. - Oesophagostomum watanabei n. sp. (Nematoda, Strongylidae) from a Japanese wild boar. J. Helminth., XXXV (1-2), 203-206.

-, 1961 b. - Systema Helminthum. Vol. III. The Nematodes of Vertebrates Inter-science Publishers New York et London.

YoRKe (W.) et MAPLESTONE (P. A.), 1926. - The Nematode parasites of Vertebrates. 536 pp., 307 fig. J. A. Churchill. London. 